\title{
AN ASYMPTOTICALLY OPTIMAL MODEL FOR ISOTROPIC HETEROGENEOUS LINEARLY ELASTIC PLATES*
}

\author{
Ferdinando Auricchio ${ }^{1}$, Carlo Lovadina ${ }^{2}$ And Alexandre L. Madureira ${ }^{3}$
}

\begin{abstract}
In this paper, we derive and analyze a Reissner-Mindlin-like model for isotropic heterogeneous linearly elastic plates. The modeling procedure is based on a Hellinger-Reissner principle, which we modify to derive consistent models. Due to the material heterogeneity, the classical polynomial profiles for the plate shear stress are replaced by more sophisticated choices, that are asymptotically correct. In the homogeneous case we recover a Reissner-Mindlin model with $5 / 6$ as shear correction factor. Asymptotic expansions are used to estimate the modeling error. We remark that our derivation is not based on asymptotic arguments only. Thus, the model obtained is more sophisticated (and accurate) than simply taking the asymptotic limit of the three dimensional problem. Moreover, we do not assume periodicity of the heterogeneities.
\end{abstract}

Mathematics Subject Classification. 35B40, 74K20.

Received: May 29, 2003. Revised: August 31, 2004.

\section{INTRODUCTION}

Laminate slender structures are often adopted in practical applications for the excellent ratio between mechanical performances and weight. However, in terms of modeling they present several challenges.

In fact, although occupying a three dimensional domain in space, slender bodies are characterized by having a one- or two-dimensional "aspect". Accordingly, dimension reduction models are posed in domains with at least one dimension less than the original problem, but the model solution should approximate as close as possible the original three-dimensional domain solution.

The modeling complexity is particularly significant for the case of slender structures made of heterogeneous materials, also in the case of two dimensional planar plate-like bodies. There have been numerous modeling attempts trying to incorporate the influence that the heterogeneity has on the solution in such problems (see for instance $[4,10]$, and references therein).

In this paper, we derive (and analyze) a version of the Reissner-Mindlin equations for the case of a plate made by isotropic heterogeneous materials. To reach this goal, we use the approach of [1] for the development

\footnotetext{
Keywords and phrases. Reissner, Mindlin, plate, heterogeneous plates, asymptotic analysis.

* This work has been partly supported by I.A.N. C.N.R. of Pavia, Italy, and also by CNPq, Brazil.

1 Dipartimento di Meccanica Strutturale, Università di Pavia, Via Ferrata 1, 27100 Pavia, Italy. e-mail: auricchio@unipv.it

2 Dipartimento di Matematica, Università di Pavia, Via Ferrata 1, 27100 Pavia, Italy. e-mail: lovadina@dimat.unipv.it

3 Departamento de Matemática Aplicada e Computacional, Laboratório Nacional de Computação Científica, Av. Getúlio Vargas 333, Petrópolis - RJ, Brazil. e-mail: alm@lncc.br
} 
of a model. Such a technique relies on a variational approach and it is based essentially on two ingredients: the choice of a variational principle and the choice of proper subspaces.

Due to the material heterogeneity, in the following we need to develop an ad hoc variational principle, which is different from the ones adopted in [1], effective only for the case of homogeneous materials. Moreover, we also have to modify the subspaces in which we look for the solution; in fact, the classical polynomial profiles for the shear stress valid for the case of homogeneous materials are now replaced by more sophisticated choices. In particular, such new profiles are derived through considerations based on asymptotic expansions.

As a result of our analysis, we obtain equations (25)-(28) defining our candidate for approximating the three dimensional heterogeneous plate problem solution.

We would like to emphasize that a plate model based on variational principles differs substantially from a model which results from considering asymptotic limits. Indeed, the former is defined by a system of singularly perturbed equations, and the corresponding solution presents a complex behavior with respect to the thickness.

On the other hand, models originated by asymptotic considerations are of a different nature. The best known example is the biharmonic plate model $(c f .[6,7,9]$ for homogeneous isotropic plates, and $[5,13]$ for the heterogeneous case). The corresponding equations do not depend singularly on the plate thickness, and usually have limited applicability, as shown in $[3,6]$.

The outline of the paper is as follows. In the next section, we describe the traditional variational approaches and why it is not possible to use them. In Section 2, we detail the variational principle upon which we base our derivation and present a description of the proposed model. Finally, in Section 3, we prove the consistency of our solution and some other convergence results. We postpone to various appendices most of the details regarding derivation of the model, asymptotic expansions, and convergence results.

Before proceeding, we introduce and explain some notation. We use one underbar for first order tensors in three variables, two underbars for second order tensors in three variables, etc. Similar notation holds with undertildes for tensors in two variables. We can then decompose 3 -vectors and $3 \times 3$ matrices as follows:

$$
\underline{u}=\left(\begin{array}{c}
u \\
\underset{u_{3}}{u}
\end{array}\right), \quad \underline{\underline{\sigma}}=\left(\begin{array}{cc}
\underset{\sim}{\sigma} & \underset{\sim}{\sim} \\
\underset{\sim}{\sigma^{T}} & \sigma_{33}
\end{array}\right) .
$$

Moreover, throughout the paper we make use of the following operators that assigns for a given tensor its moments:

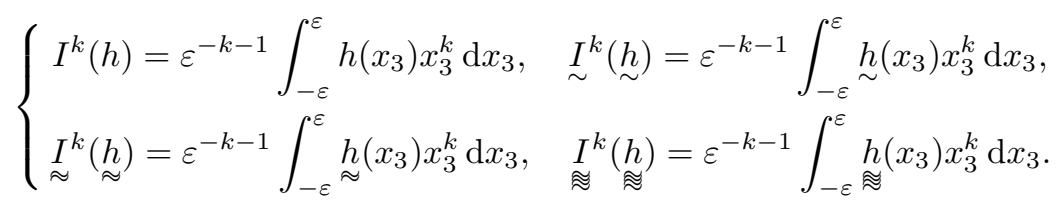

\section{Plate model construction by variational approach}

We consider a linearly elastic body occupying the three-dimensional domain $P^{\varepsilon}=\Omega \times(-\varepsilon, \varepsilon)$, where $\Omega$ is a bounded two-dimensional domain. Clamped on the lateral boundary $\partial P_{L}^{\varepsilon}=\partial \Omega \times(-\varepsilon, \varepsilon)$, the body is under the action of a surface force density $\underline{g}^{\varepsilon}$ on its top and bottom $\partial P_{ \pm}^{\varepsilon}=\Omega \times\{-\varepsilon, \varepsilon\}$, and a volume force density $f^{\varepsilon}$. The equations of linear elasticity state that the displacement $\underline{u}^{\varepsilon}: P^{\varepsilon} \rightarrow \mathbb{R}^{3}$, and the stress $\underline{\sigma}^{\varepsilon}: P^{\varepsilon} \rightarrow \mathbb{R}_{\mathrm{sym}}^{3 \times 3}$ satisfy

$$
\left\{\begin{array}{lr}
A \sigma^{\varepsilon}=\underline{e}\left(\underline{u}^{\varepsilon}\right), & -\operatorname{div} \underline{\sigma}^{\varepsilon}=\underline{f}^{\varepsilon} \quad \text { in } P^{\varepsilon}, \\
\underline{u}^{\varepsilon}=0 \text { on } \partial P_{L}^{\varepsilon}, & \underline{\sigma}^{\varepsilon} \underline{n}=\underline{g}^{\varepsilon} \text { on } \partial P_{ \pm}^{\varepsilon},
\end{array}\right.
$$

where $\underline{\underline{e}}\left(\underline{u}^{\varepsilon}\right)=\left(\underline{\underline{\nabla}} \underline{u}^{\varepsilon}+\underline{\underline{\nabla}}^{T} \underline{u}^{\varepsilon}\right) / 2$ is the symmetric part of the gradient of $\underline{u}^{\varepsilon}$, and $A$ is the compliance tensor. Limiting the discussion to an isotropic material, the compliance tensor $A$ is defined as

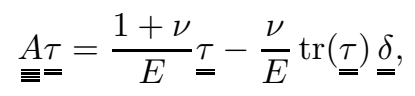


for every second-order tensor $\tau$, being $\delta$ the identity tensor. The Young's modulus $E$ and the Poisson's ratio $\nu$ are material parameters, which might $\overline{\bar{d}}$ epend on the transverse variable $x_{3}$, being however independent of the planar variable $\underset{\sim}{x}$.

A way to systematically derive two dimensional plate models is the variational approach proposed in [1]. This procedure consists in choosing a variational principle, which corresponds to a weak formulation of (1). The plate model is then derived restricting the test and trial spaces to suitable subspaces.

In [1] the following two different variational principles were considered to treat the case of isotropic and homogeneous bodies.

- The couple $\left(\underline{u}^{\varepsilon}, \underline{\underline{\sigma^{\varepsilon}}}\right)$, solution of Problem (1), is the unique critical point of the functional

$$
J(\underline{v}, \underline{\underline{\tau}})=\frac{1}{2} \int_{P^{\varepsilon}} \underline{\underline{\underline{\underline{A}}}} \underline{\underline{\tau}}: \underline{\underline{\tau}} \mathrm{d} \underline{\mathrm{d}} \underline{x}+\int_{P^{\varepsilon}} \underline{v} \cdot \underline{\operatorname{div}} \underline{\underline{\tau}} \mathrm{d} \underline{x}+\int_{P^{\varepsilon}} \underline{f} \cdot \underline{v} \mathrm{~d} \underline{x}
$$

on $\underline{L}^{2}\left(P^{\varepsilon}\right) \times \underline{\underline{\Sigma}}_{\underline{g}^{\varepsilon}}$, where $\underline{\underline{\underline{\Sigma}}}_{\underline{\underline{g}}}=\left\{\underline{\underline{\sigma}}: \underline{\underline{\sigma}} \in \underline{\underline{H}}\left(\underline{\operatorname{div}}, P^{\varepsilon}\right), \underline{\underline{\sigma}} \underline{\underline{a}}=\underline{g}^{\varepsilon}\right.$ on $\left.\partial P_{ \pm}^{\varepsilon}\right\}$.

Following the variational approach of $\overline{\overline{1}}$ 1], plate models are derived restricting (3) to particular subspaces of $\underline{L}^{2}\left(P^{\varepsilon}\right) \times \underline{\Sigma}_{g^{\varepsilon}}$, characterized by having specific polynomial dependences in the transverse direction.

It is interesting to notice that not all choices of subspaces lead to a well-posed PDE system, as well as some specific choices lead to models that, although well-posed, are divergent in a sense that we make clear later on. It is also interesting to recall that, starting from functional (3) and adopting two different subspaces, it is possible to obtain two versions of the Reissner-Mindlin model, both with the shear correction factor $5 / 6$. By "version", we mean that the left hand side of the equations are the same as the standard Reissner-Mindlin ones, up to the shear correction factor, while the right hand side may differ.

- The couple $\left(\underline{u}^{\varepsilon}, \underline{\sigma^{\varepsilon}}\right)$, solution of Problem (1), is the unique critical point of the functional

$$
J^{*}(\underline{v}, \underline{\underline{\tau}})=\frac{1}{2} \int_{P^{\varepsilon}} \underline{\underline{\underline{A}}} \underline{\underline{\tau}}: \underline{\underline{\tau}} \mathrm{d} \underline{\mathrm{x}}-\int_{P^{\varepsilon}} \underline{\underline{e}}(\underline{v}): \underline{\underline{\tau}} \mathrm{d} \underline{x}+\int_{P^{\varepsilon}} \underline{f} \cdot \underline{v} \mathrm{~d} \underline{x}+\int_{\partial P_{ \pm}^{\varepsilon}} \underline{g} \cdot \underline{v} \mathrm{~d} \underset{\sim}{\sim}
$$

on $\left\{\underline{v} \in \underline{H}^{1}\left(P^{\varepsilon}\right): \underline{v}=0\right.$ on $\left.\partial P_{\mathrm{L}}^{\varepsilon}\right\} \times L^{2}\left(P^{\varepsilon}\right)$.

Again, modeling is possible by searching for critical points in subspaces with certain polynomial dependence. It is interesting to notice that the minimum energy models are a particular instance of this approach, and that the simplest model derived using this approach is not a minimum energy model.

Finally, we recall that starting from the functional (4) and adopting specific subspaces, the resulting system is a variant of Reissner-Mindlin model, with shear correction factor 1. The other models based on (4) are either more complicate than Reissner-Mindlin or divergent.

\subsection{Definition of model consistency}

As discussed in the introduction, every plate model is supposed to approximate the 3D solution for the limiting "thin" plate case. Accordingly, we say that a plate model is consistent, or convergent, if for every "reasonable" choice of loads it holds

$$
\lim _{\varepsilon \rightarrow 0} \frac{\left\|\underline{u}^{\varepsilon}-\underline{u}^{M, \varepsilon}\right\|_{H^{1}\left(P^{\varepsilon}\right)}}{\left\|\underline{u}^{\varepsilon}\right\|_{H^{1}\left(P^{\varepsilon}\right)}}=0,
$$

where $u^{\varepsilon}$ are the $3 \mathrm{D}$ displacements and $u^{M, \varepsilon}$ are the model displacements.

More precisely, let us suppose that there exist $\varepsilon$-independent loads $\underline{f}$ and $\underline{g}$ defined as $\underline{f}: \Omega \times(-1,1) \rightarrow \mathbb{R}^{3}$, $\underline{g}: \Omega \times\{-1,1\} \rightarrow \mathbb{R}^{3}$ and such that

$$
\begin{cases}\left.\underset{\sim}{f(x}, \hat{x}_{3}\right)=\varepsilon^{-1}{\underset{\sim}{\varepsilon}}^{\varepsilon}\left(\underset{\sim}{x}, \varepsilon \hat{x}_{3}\right), & f_{3}\left(\underset{\sim}{x}, \hat{x}_{3}\right)=\varepsilon^{-2} f_{3}^{\varepsilon}\left(\underset{\sim}{x}, \varepsilon \hat{x}_{3}\right), \\ \left.\underset{\sim}{g} \underset{\sim}{x}, \hat{x}_{3}\right)=\varepsilon^{-2}{\underset{\sim}{g}}^{\varepsilon}\left(\underset{\sim}{x}, \varepsilon \hat{x}_{3}\right), & \left.g_{3}\left(\underset{\sim}{x}, \hat{x}_{3}\right)=\varepsilon^{-3} g_{3}^{\varepsilon} \underset{\sim}{\sim}, \varepsilon \hat{x}_{3}\right),\end{cases}
$$


for $\hat{x}_{3} \in(-1,1)$. Also, let us assume that the functions

$$
\hat{\nu}\left(\hat{x}_{3}\right)=\nu\left(\varepsilon \hat{x}_{3}\right), \quad \hat{E}\left(\hat{x}_{3}\right)=E\left(\varepsilon \hat{x}_{3}\right) \quad \text { for } \hat{x}_{3} \in(-1,1),
$$

are independent of $\varepsilon$ and, since there is no risk of confusion, we still write $\nu$ and $E$, even in the domain $(-1,1)$. Under these hypotheses, as detailed in Appendix B, $\underline{u}^{\varepsilon}$ converges asymptotically to

$$
\underline{u}_{L}(\underline{x})=\left(\begin{array}{c}
\varepsilon \zeta_{\sim}^{1}(\underset{\sim}{x})-x_{3} \underset{\sim}{\nabla} \zeta_{3}^{0}(\underset{\sim}{x}) \\
\underbrace{x}_{\sim})
\end{array}\right)
$$

where $\zeta^{1}$ and $\zeta_{3}^{0}$ solve the following system of equations

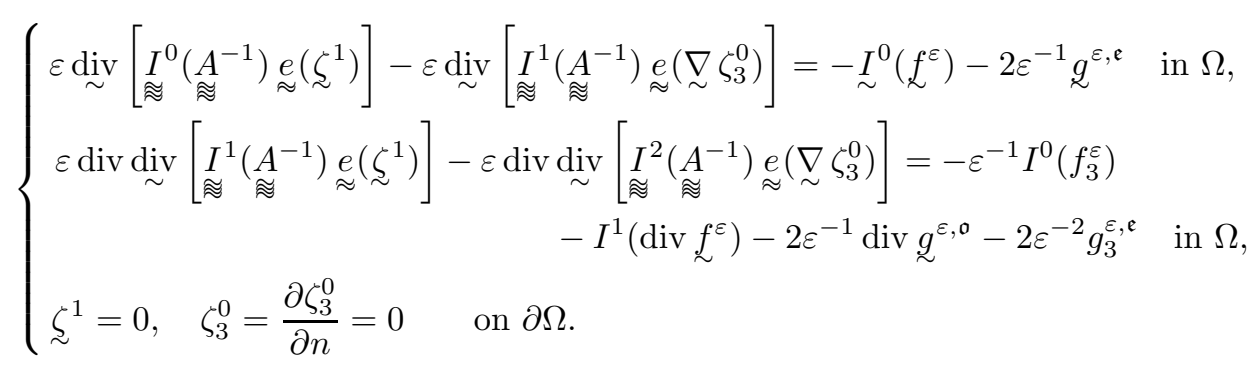

with $\underset{\approx}{\approx}$ defined as

$$
\underset{\approx \approx}{A \tau}=\frac{(1+\nu)}{E} \tau-\frac{\nu}{E} \operatorname{tr} \underset{\approx}{\approx} \underset{\approx}{\delta}
$$

and with the even and odd parts of $\underline{g}$ defined as

$$
\underline{g}^{\varepsilon, \mathfrak{e}}(\underset{\sim}{x})=\frac{1}{2}\left[\underline{g}^{\varepsilon}(\underset{\sim}{x}, \varepsilon)+\underline{g}^{\varepsilon}(\underset{\sim}{x},-\varepsilon)\right], \quad \underline{g}^{\varepsilon, \mathfrak{o}}(\underset{\sim}{x})=\frac{1}{2}\left[\underline{g}^{\varepsilon}(\underset{\sim}{x}, \varepsilon)-\underline{g}^{\varepsilon}(\underset{\sim}{x},-\varepsilon)\right] .
$$

We say that the plate model is consistent, or convergent, if condition (5) is satisfied for all loads such that at least one of the functions $\zeta^{1}$ and $\zeta_{3}^{0}$ are nonzero.

Remark 1.1. Note that [12] uses a slightly different definition of consistency.

Remark 1.2. Note that (7) couples the membrane and bending problems. Nevertheless, if $\nu$ and $E$ are even functions (symmetric plates), then it is easy to see that (7) decouples into the membrane equation for $\zeta^{1}$, and the biharmonic equation for $\zeta_{3}^{0}$.

\subsection{Problems with the Hellinger-Reissner principle}

Taking into account the previous considerations, it would be natural to choose functional (3) as a starting point to derive a plate model also for the case of heterogeneous materials. Unfortunately, the direct application of functional (3) is not possible since, due to the heterogeneity, it leads to divergent models, as discussed below.

Let us consider the case where $g_{3}=0$ and $\nu$ and $E$ are even functions of $x_{3}$. Since the three-dimensional problem (1) decouples in bending and membrane equations, for the sake of simplicity we consider only the membrane contribution.

The simplest model based on (3) is obtained by assuming that trial/test in-plane displacements and in-plane stresses are constant along the thickness ( $c f$. [1] for the details)

$$
\underline{u}^{M}(\underline{x})=(\underset{\underset{\sim}{\eta}(\underset{x}{x})}{0}), \quad{\underset{\sim}{\sigma}}^{M}(\underline{x})=\underset{\sim}{\sigma}(x), \quad \sigma_{33}^{M}(\underline{x})=0, \quad{\underset{\sim}{\sigma}}^{M}(\underline{x})=\varepsilon^{-1} x_{3} \underline{q}^{\varepsilon, \mathfrak{e}}(\underset{\sim}{x}),
$$


for some $\underset{\sim}{\eta}$ and $\underset{\sim}{\sigma}$. Searching now for critical points, we obtain the following membrane equation

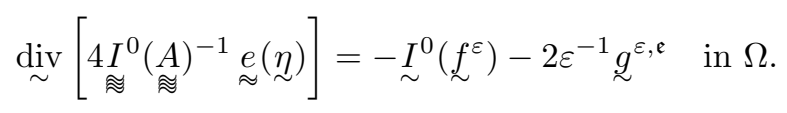

On the other hand, for this specific (uncoupled) problem the 3D in-plane displacement field $u_{\sim}^{\varepsilon}$ converges asymptotically to $\varepsilon \zeta^{1}$, solution of $(c f .(7))$

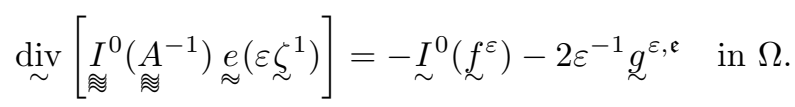

Hence, the obtained membrane model is divergent since for a general heterogeneous material

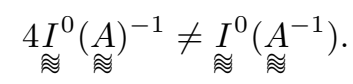

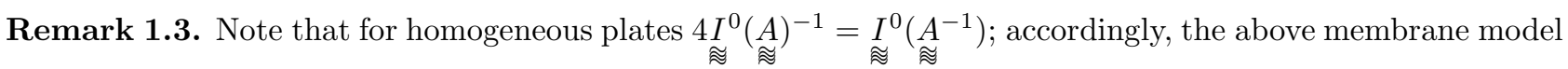
is consistent, since $\underset{\sim}{u} u^{M}=\underset{\sim}{\eta}=\varepsilon \zeta^{1}$.

To summarize, the extension of the techniques detailed in [1] to the case of heterogeneous plates leads to the following two difficulties:

- the variational principle (3) is no longer suitable;

- polynomial profiles in the transverse direction are no more satisfactory.

In particular, the second point is due to the fact that now the profiles for the stress tensor components have a complicated shape, depending on the material heterogeneities. Accordingly, assuming polynomial profiles for all the unknowns may lead to a poor representation of the stress tensor in the transverse direction.

In what follows we overcome these difficulties by:

- choosing a different variational principle;

- choosing a subspace for the stress tensor which takes into account the heterogeneity of the plate.

\section{A NEW MODEL}

\subsection{A new functional}

As shown in equation (13), the processes of inverting and homogenizing do not commute in the case of heterogeneous tensors, yielding an incorrect constitutive relation for the plate planar components. To overcome this difficulty, we try to impose directly in the model the following planar constitutive equation

$$
\underset{\approx}{\sigma^{\varepsilon}}=\underset{\approx}{A^{-1}} \underset{\approx}{\underset{\sim}{e}}\left(u^{\varepsilon}\right)+\frac{\nu}{E} \sigma_{33}^{\varepsilon} \underset{\approx}{A^{-1}} \underset{\approx}{\delta} .
$$

To reach this goal, we first introduce the space $\underline{\underline{S}}_{\underline{g^{\varepsilon}}}$, defined by

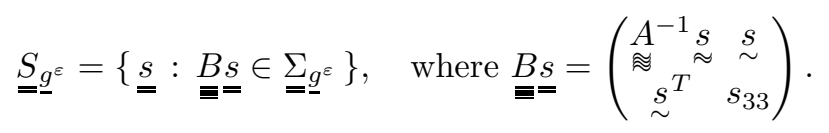

We then consider the new functional

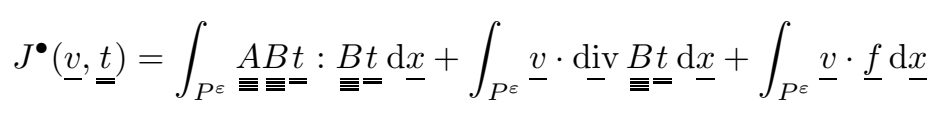


defined on $\underline{L}^{2}\left(P^{\varepsilon}\right) \times \underline{S}_{g^{\varepsilon}}$. It is easily seen that $\left(\underline{u}^{\varepsilon}, \underline{\underline{\sigma}}^{\varepsilon}\right) \in \underline{L}^{2}\left(P^{\varepsilon}\right) \times \underline{\underline{S}}_{\underline{g}^{\varepsilon}}$ is the unique critical point of functional (3), if and only if the couple $\left(\underline{u}^{\varepsilon}, \underline{\underline{s}}^{\varepsilon}\right)=\left(\underline{u}^{\varepsilon}, B^{-1} \underline{\underline{\sigma}}^{\bar{\varepsilon}}\right) \in \underline{L}^{2}\left(\bar{P}^{\varepsilon}\right) \times \underline{\underline{S}}_{g^{\varepsilon}}$ is the unique critical point of functional (16). Since $\underline{\underline{s}}^{\varepsilon}=\underline{\underline{\underline{\underline{B}}}}^{-1}{\underline{\underline{\sigma^{\varepsilon}}}}^{\varepsilon}$, it follows that it holds $(c f .(15))$

$$
\underset{\sim}{s^{\varepsilon}}=\underset{\sim}{e}\left(u^{\varepsilon}\right)+\frac{\nu}{E} \sigma_{33}^{\varepsilon} \underset{\sim}{\delta}=\underset{\approx}{A \sigma^{\varepsilon}}, \quad \underset{\sim}{s^{\varepsilon}}=\underset{\sim}{\sigma^{\varepsilon}}, \quad s_{33}^{\varepsilon}=\sigma_{33}^{\varepsilon} .
$$

Moreover, we have that $\left(\underline{u}^{\varepsilon}, \underline{\underline{s}}^{\varepsilon}\right) \in \underline{L}^{2}\left(P^{\varepsilon}\right) \times \underline{\underline{S}}_{\underline{g}^{\varepsilon}}$ satisfies the weak equations

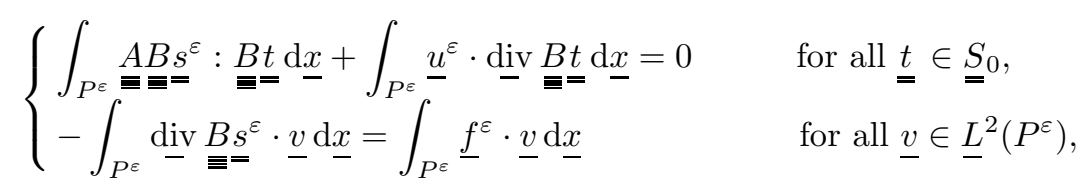

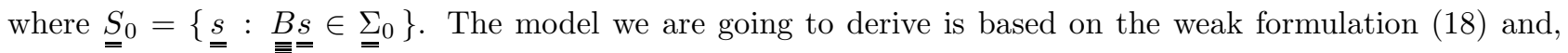
similarly to [1], it will be obtained by looking for critical points of $J^{\bullet}$ within suitable subspaces $\underline{\hat{L}}^{2}\left(P^{\varepsilon}\right) \times \underline{\underline{S}}_{\underline{S}^{\varepsilon}} \subset$ $\underline{L}^{2}\left(P^{\varepsilon}\right) \times \underline{S}_{g^{\varepsilon}}$.

Remark 2.1. We notice that Auricchio and Sacco in [4] already used the constitutive equation (14) to develop their model.

\subsection{Subspace choice}

Our choices of profiles for displacements and stresses are based on the asymptotic analysis of the threedimensional solution. To obtain the simplest possible convergent model, we decided to exclude the profile of the asymptotic limit of $\sigma_{33}^{\varepsilon}$. As we describe in Appendix B,

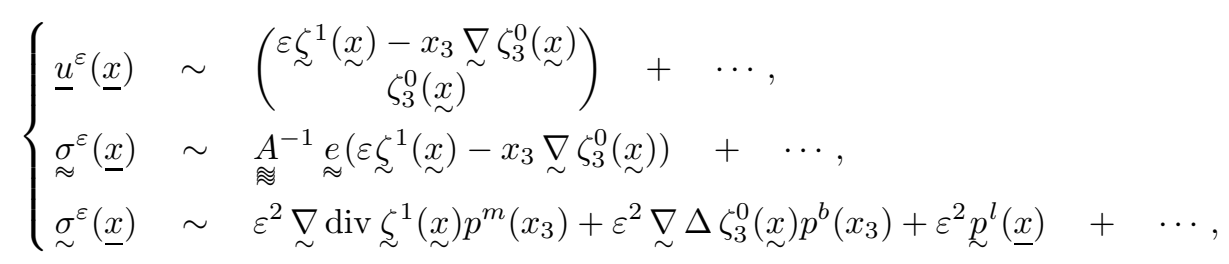

where $\zeta^{1}$ and $\zeta_{3}^{0}$ solve (7), and

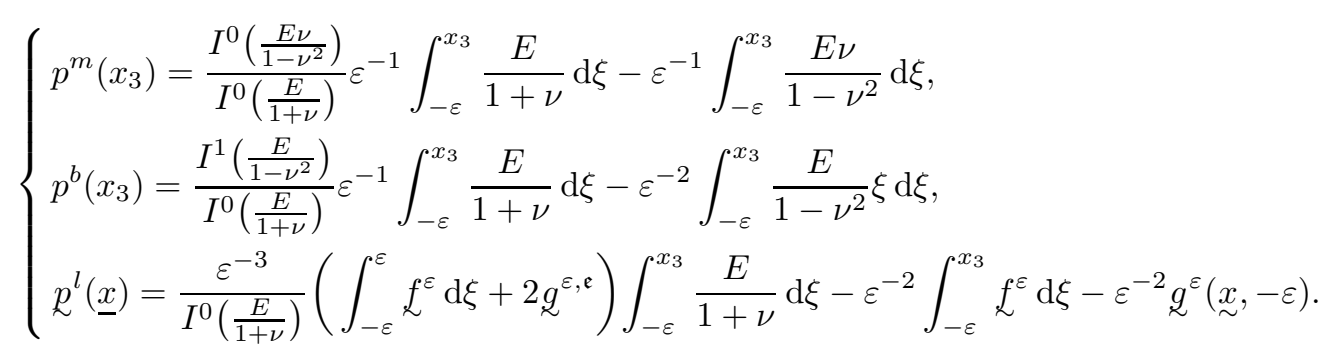

Remark 2.2. Note that $p^{m}( \pm \varepsilon)=p^{b}( \pm \varepsilon)=0$. Moreover, $\left.\varepsilon^{2} \underline{\sim}^{l} \underset{\sim}{x}, \varepsilon\right)=\underline{\sim}^{\varepsilon}(\underset{\sim}{x}, \varepsilon)$ and $\varepsilon^{2} p^{l}(\underset{\sim}{x},-\varepsilon)=-g_{\sim}^{\varepsilon}(\underset{\sim}{x},-\varepsilon)$. Moreover, ${\underset{\sim}{l}}^{l}$ depends only on the load. 
Remark 2.3. We remark that the leading terms of the variables involved in (20) have the following shapes in the transverse direction:

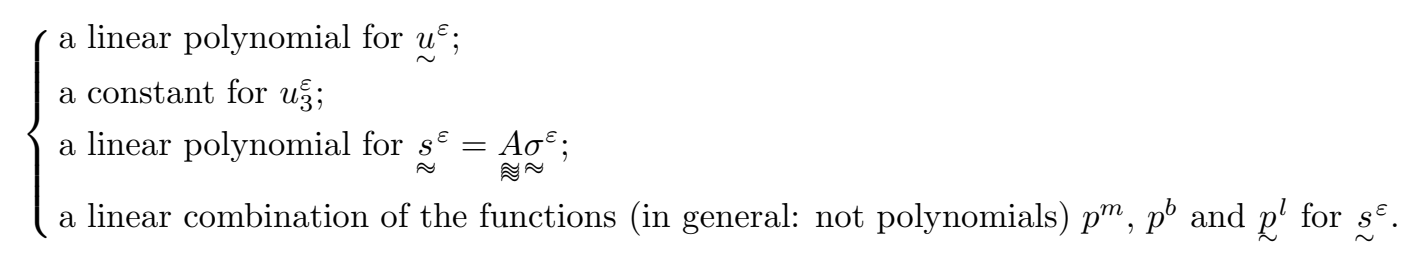

We are now ready to introduce the subspaces $\underline{\hat{L}}^{2}\left(P^{\varepsilon}\right) \subset \underline{L}^{2}\left(P^{\varepsilon}\right)$ and $\underline{\underline{S}}_{\underline{g}^{\varepsilon}} \subset \underline{\underline{S}}_{\underline{g}^{\varepsilon}}$, respectively for the displacement and the stress-related unknowns, by setting

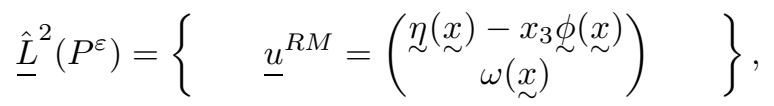

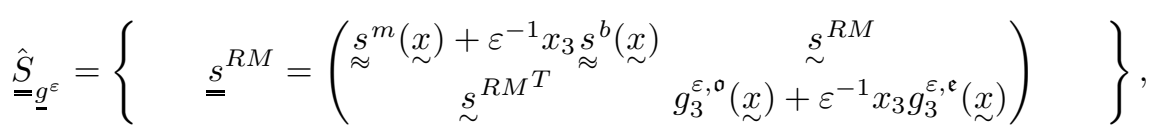

where $\underset{\sim}{\eta} \underset{\sim}{\phi}, \omega, \underset{\sim}{s}$ and $\underset{\sim}{s}$ are functions defined on $\Omega$.

Moreover, for the shear stress ${\underset{\sim}{s}}^{R M}$ there are two possibilities, since $p^{m}$ and $p^{b}$ can be linearly dependent. This occurs for instance, if $\nu$ is constant, and consequently $p^{m}=0$. Hence, we assume that

$$
\underline{\sim}^{R M}(\underline{x})=\varepsilon^{2} \underline{\sim}^{l}(\underline{x})+ \begin{cases}\underset{\sim}{s}(\underset{\sim}{x}) p^{m}\left(x_{3}\right)+{\underset{\sim}{s}}^{b}(\underset{\sim}{x}) p^{b}\left(x_{3}\right) & \text { if } p^{m}, p^{b} \text { are lin. indep. } \\ \underset{\sim}{s^{b}}(\underset{\sim}{x}) p^{b}\left(x_{3}\right) & \text { otherwise. }\end{cases}
$$

\subsection{Model derivation}

Restricting the test and trial spaces as discussed in subsection 2.2, problem (18) reduces to find $\left(\underline{u}^{R M}, \underline{\underline{s}}^{R M}\right) \in$ $\underline{\hat{L}}^{2}\left(P^{\varepsilon}\right) \times \underline{\underline{S}}_{\underline{g^{\varepsilon}}}$ solution of

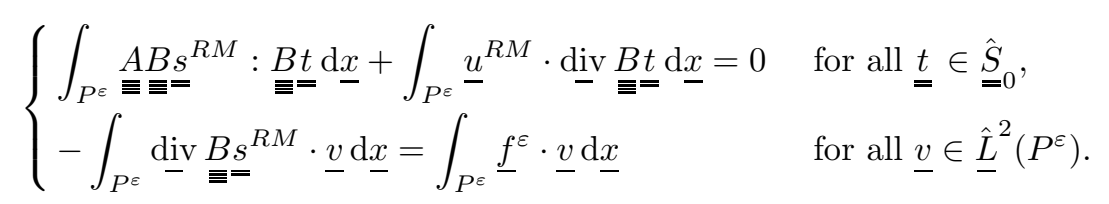

Due to the structure of $\underline{\underline{L}}^{2}\left(P^{\varepsilon}\right)$ and $\underline{\underline{S}}_{g^{\varepsilon}}$, the above system (24) can be seen as a system whose unknowns are the following functions, all defined on the mid-plane $\Omega$ :

$$
\left\{\begin{array}{l}
\text { the unknowns } \underset{\sim}{\eta}, \underset{\sim}{\phi} \text { and } \omega \text { related to the kinematic fields; } \\
\text { the unknowns } \underset{\sim}{\underset{\sim}{s}} \text { and } \underset{\sim}{s^{b}} \text { related to in-plane stress; } \\
\text { the unknowns } \underset{\sim}{s} \text { and } \underset{\sim}{s} \text { related to shear stress. }
\end{array}\right.
$$

In particular, our approximation to $\underline{u}^{\varepsilon}$ is given by

$$
\underline{u}^{R M}=(\underset{\sim}{\underset{\sim}{\eta}(\underset{\sim}{(x)})} \underset{\sim}{\sim} \underset{\sim}{\phi(x)}) .
$$


From the weak formulation (24) it is possible to show that $\underset{\sim}{\eta}, \underset{\sim}{\phi}$ and $\omega$ are uniquely determined by the following system of partial differential equations on $\Omega$ :

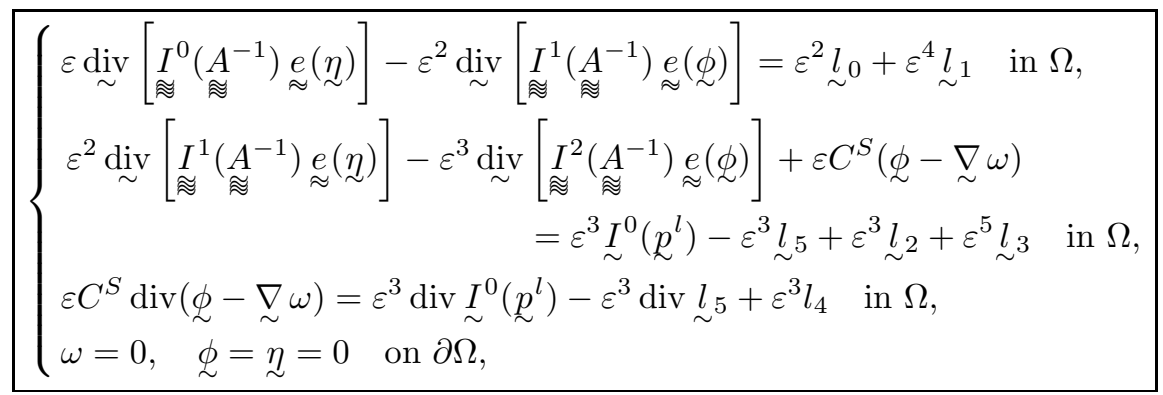

where

$$
\begin{aligned}
& C^{S}=\left(I^{0}\left(p^{m}\right) I^{0}\left(p^{b}\right)\right) \underset{\approx}{M}\left(\begin{array}{cc}
I^{0}\left(p^{m}\right) \\
I^{0}\left(p^{b}\right)
\end{array}\right), \quad \underset{\approx}{M} \begin{cases}\left(\begin{array}{ll}
d_{11} & d_{12} \\
d_{12} & d_{22}
\end{array}\right)^{-1} & \text { if } d_{11} d_{22}-\left(d_{12}\right)^{2} \neq 0, \\
\frac{1}{d_{22}}\left(\begin{array}{ll}
0 & 0 \\
0 & 1
\end{array}\right)^{-} & \text {otherwise, }\end{cases} \\
& d_{11}=I^{0}\left(\frac{2(1+\nu)}{E}\left(p^{m}\right)^{2}\right), \quad d_{12}=I^{0}\left(\frac{2(1+\nu)}{E} p^{m} p^{b}\right), \quad d_{22}=I^{0}\left(\frac{2(1+\nu)}{E}\left(p^{b}\right)^{2}\right) .
\end{aligned}
$$

The loads are given by

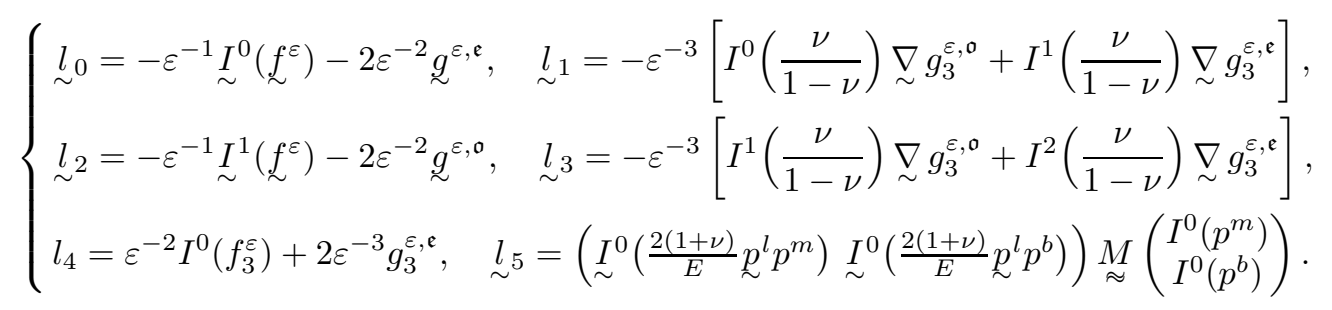

Details relative to the computations to go from (24) to (26) can be found in Appendix A.

Remark 2.4. Note that the definitions of the $l_{i}$ s are such that they are independent of $\varepsilon$ if the scaling (6) holds.

After $\underset{\sim}{\eta}, \underset{\sim}{\phi}$, and $\omega$ have been determined, the computation of $\underset{\sim}{s}{ }^{R M}, \underset{\sim}{s^{R M}}$, and $s_{33}^{R M}$ can be recovered as a post-processing. In particular the fields $\underset{\sim}{\underset{\sim}{m}}$ and $\underset{\sim}{s}$ are the solution of the algebraic system

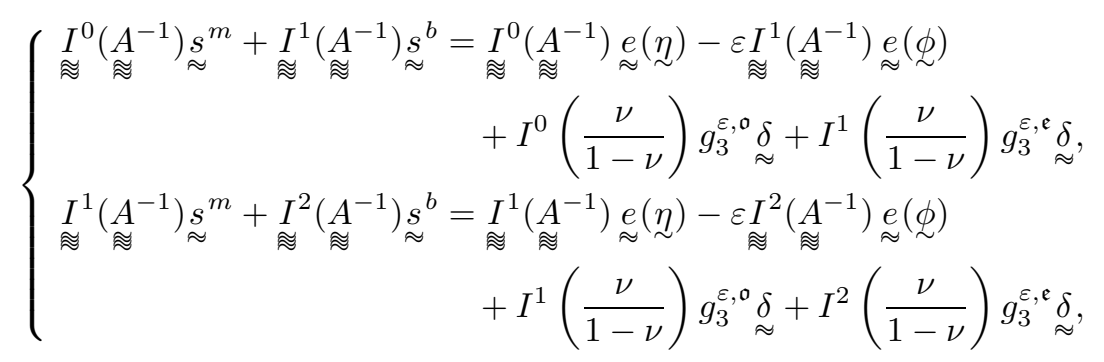


while the fields $\underset{\sim}{s}$ and $\underset{\sim}{s}$ are computed as

$$
\left({\underset{\sim}{s}}^{m}{\underset{\sim}{s}}^{b}\right)=(-\underset{\sim}{\phi}+\underset{\sim}{\nabla} \omega)\left(I^{0}\left(p^{m}\right) I^{0}\left(p^{b}\right)\right) \underset{\approx}{M}-\varepsilon^{2}\left(\underset{\sim}{I^{0}}\left(\frac{2(1+\nu)}{E} \underset{\sim}{p} p^{l}\right) \underset{\sim}{I^{0}}\left(\frac{2(1+\nu)}{E}{\underset{\sim}{p}}^{l} p^{b}\right)\right) \underset{\approx}{M} .
$$

Finally, the stress components are given by $(c f .(17))$

$$
{\underset{\approx}{\sigma}}^{R M}=\underset{\approx}{A^{-1}} \underset{\approx}{s}{ }^{R M}+\frac{\nu}{1-\nu} \sigma_{33}^{R M} \underset{\approx}{\delta}, \quad \sigma^{R M}={\underset{\sim}{s}}^{R M}, \quad \sigma_{33}^{R M}=g_{3}^{\varepsilon, \mathfrak{o}}(\underset{\sim}{x})+\varepsilon^{-1} x_{3} g_{3}^{\varepsilon, \mathfrak{e}}(\underset{\sim}{x}) .
$$

\section{Homogeneous materials}

When the plate is composed of homogeneous material, the equations (26)-(30) considerably simplify. Since the material functions $\nu$ and $E$ are symmetric, the equations decouple into membrane and bending parts. The membrane equation reduces to

$$
\left\{\begin{array}{l}
-2 \varepsilon \underset{\sim}{\operatorname{div}} \underset{\approx}{\approx} \underset{\approx}{\approx} \underset{\sim}{\approx}(\eta)=\varepsilon{\underset{\sim}{I}}^{0}\left(f^{\varepsilon}\right)+2 \underline{\sim}^{\varepsilon, \mathfrak{e}}+2 \varepsilon \frac{\nu}{1-\nu} \underset{\sim}{\nabla} g_{3}^{\varepsilon, \mathfrak{o}} \quad \text { in } \Omega, \\
\eta=0 \text { on } \partial \Omega .
\end{array}\right.
$$

Equations (32) are the same as derived in [1], although the two derivations are based on different choices of spaces.

For the bending equation, we have

$$
\left\{\begin{array}{l}
-\frac{2 \varepsilon^{3}}{3} \operatorname{div}_{\sim} \underset{\approx}{A^{-1}} \underset{\approx}{e}(\phi)+\varepsilon \frac{5}{6} \frac{E}{1+\nu}(\phi-\underset{\sim}{\nabla} \omega)=-\varepsilon^{3}{\underset{\sim}{\sim}}_{5}-\varepsilon^{2} \frac{2 \nu}{3(1-\nu)} \underset{\sim}{\nabla} g_{3}^{\varepsilon, \mathfrak{e}} \quad \text { in } \Omega, \\
\varepsilon \frac{5}{6} \frac{E}{1+\nu} \operatorname{div}(\underset{\sim}{\phi}-\underset{\sim}{\nabla} \omega)=F+2 g_{3}^{\varepsilon, \mathfrak{e}}+\frac{\varepsilon}{3} \operatorname{div} \underset{\sim}{g^{\varepsilon, \mathfrak{o}}} \quad \text { in } \Omega, \\
\omega=0, \quad \phi=0 \quad \text { on } \partial \Omega .
\end{array}\right.
$$

The loads are given by

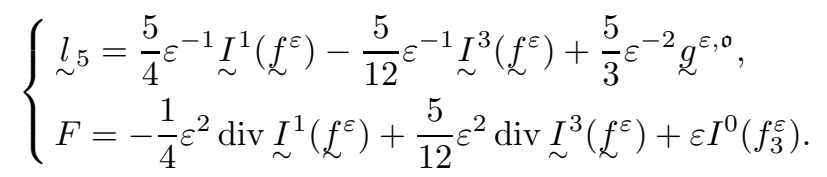

The problem defined by (33)-(34) differs from the bending problem found in [1]. Indeed, the right hand side of the (33) is new, and it incorporates higher order moments of $\underset{\sim}{f}$. Notice also that we recover a model of Reissner-Mindlin type, with shear correction factor $5 / 6$.

\section{Model COnsistency And some COnVERGEnCE Results}

\subsection{Model consistency}

We now study the consistency of our model, by performing an asymptotic expansion with respect to $\varepsilon$ for both the 3D displacement solution and the model displacement solution. The key point for proving that (cf. (5))

$$
\lim _{\varepsilon \rightarrow 0} \frac{\left\|\underline{u}^{\varepsilon}-\underline{u}^{R M}\right\|_{H^{1}\left(P^{\varepsilon}\right)}}{\left\|\underline{u}^{\varepsilon}\right\|_{H^{1}\left(P^{\varepsilon}\right)}}=0
$$

is to recognize that the leading terms of both the expansions coincide. More precisely, as shown in Appendix B, the $3 \mathrm{D}$ displacement solution $\underline{\underline{u}} \underline{\varepsilon}(\underline{x})$ admits the following expansion

$$
\underline{u}^{\varepsilon}(\underline{x})=\underline{u}_{L}(\underline{x})+\text { higher order terms }
$$


where

$$
\underline{u}_{L}(\underline{x})=\left(\begin{array}{c}
\varepsilon \underbrace{\zeta^{1}}_{\sim} \underset{\sim}{x})-x_{3} \underset{\sim}{\nabla} \zeta_{3}^{0}(\underset{\sim}{(x)}(\underset{\sim}{x}) \\
\\
\end{array}\right.
$$

On the other hand ( $c f$. Appendix C), the model displacement solution $\underline{u}^{R M}(\underline{x})$ can be written as

$$
\underline{u}^{R M}(\underline{x})=\underline{u}_{L}^{R M}(\underline{x})+\text { higher order terms }
$$

where

$$
\underline{u}_{L}^{R M}(\underline{x})=\left(\underset{\underset{\eta^{1}}{\eta^{1}}(\underset{\sim}{x})-x_{3} \underline{\phi}^{0}(\underset{\sim}{x})}{\left.\underset{\omega^{0}}{x}\right)}\right.
$$

We have the following result.

Lemma 3.1. For the leading terms $\underline{u}_{L}$ and $\underline{u}_{L}^{R M}$ defined by (37) and (39), it holds

$$
\underline{u}_{L}=\underline{u}_{L}^{R M} .
$$

Proof. From (59) of Appendix B, we have that the terms $\zeta^{1}$ and $\zeta_{3}^{0}$ involved in $\underline{u}_{L}(\underline{x})(c f .(37))$ are uniquely determined by

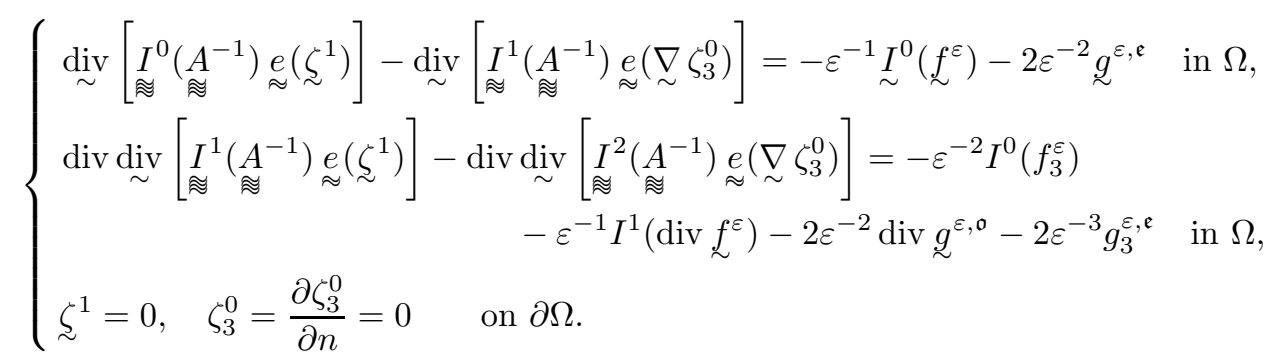

On the other hand, from (74) of Appendix C, we have that ${\underset{\sim}{1}}^{1}, \phi^{0}, \omega^{0} \in H_{0}^{1}(\Omega)$ solve

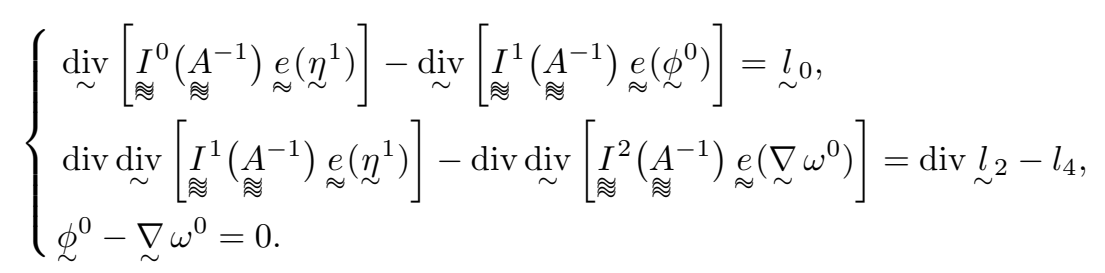

Hence, $\phi^{0}=\underset{\sim}{\nabla} \omega^{0}$ and $\partial \omega^{0} / \partial n=0$, so that $\eta_{\sim}^{1}$ and $\omega^{0}$ are determined by

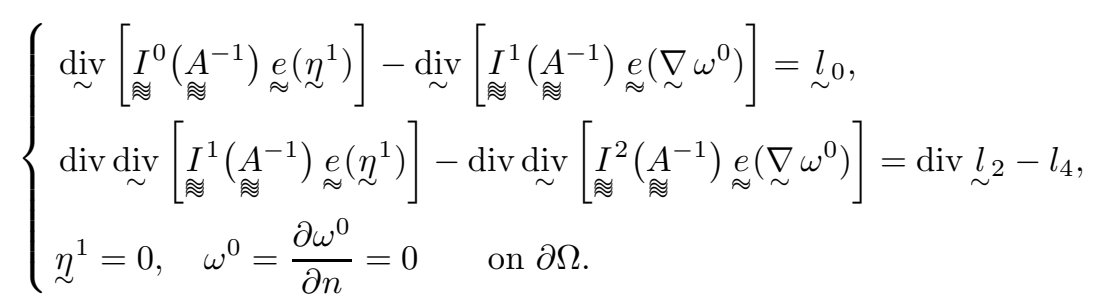

Recalling (28), we see that system (40) is the same as (42). It follows that $\zeta^{1}=\eta^{1}$ and $\zeta_{3}^{0}=\omega^{0}$, so that $\underline{u}_{L}=\underline{u}_{L}^{R M}$. 
In the result below, we prove the consistency of our model. To be able to obtain relative convergence estimates, we shall assume that that either $\zeta_{3}^{0} \neq 0$ or $\zeta^{1} \neq 0$. We shall actually assume this hypothesis throughout the paper.

Theorem 3.2. Let $\underline{u}^{\varepsilon}$ and $\underline{u}^{R M}$ be defined by (1), and (25)-(28). Then there exists a constant $C=C(\Omega, \underline{f}, \underline{g})$ independent of $\varepsilon$, such that

$$
\frac{\left\|\underline{u}^{\varepsilon}-\underline{u}^{R M}\right\|_{H^{1}\left(P^{\varepsilon}\right)}}{\left\|\underline{u}^{\varepsilon}\right\|_{H^{1}\left(P^{\varepsilon}\right)}} \leq C \varepsilon
$$

Proof. In this proof, we consider only the case when the third component of $\underline{u}_{L}$, i.e. $\zeta_{3}^{0}$, is not identically zero. The case when $\zeta_{3}^{0}=0$ but $\zeta^{1} \neq 0$ can be handled with the same technique. Since $\underline{u}_{L}=\underline{u}_{L}^{R M}$, from the triangle inequality it holds

$$
\frac{\left\|\underline{u}^{\varepsilon}-\underline{u}^{R M}\right\|_{H^{1}\left(P^{\varepsilon}\right)}}{\left\|\underline{u}^{\varepsilon}\right\|_{H^{1}\left(P^{\varepsilon}\right)}} \leq \frac{\left\|\underline{u}^{\varepsilon}-\underline{u}_{L}\right\|_{H^{1}\left(P^{\varepsilon}\right)}+\left\|\underline{u}_{L}^{R M}-\underline{u}^{R M}\right\|_{H^{1}\left(P^{\varepsilon}\right)}}{\left\|\underline{u}^{\varepsilon}\right\|_{H^{1}\left(P^{\varepsilon}\right)}} .
$$

From Theorem B.1 of Appendix B it follows

$$
\left\|\underline{u}^{\varepsilon}-\underline{u}_{L}\right\|_{H^{1}\left(P^{\varepsilon}\right)} \leq c \varepsilon^{3 / 2} .
$$

On the other hand, since

$$
\underline{u}_{L}^{R M}(\underline{x})-\underline{u}^{R M}=(\underbrace{\left(\varepsilon \eta^{1}(\underset{\sim}{x})-\underset{\omega^{0}(\underset{\sim}{\eta})-\omega(\underset{\sim}{x})}{(x)}\right)-x_{3}\left(\underset{\sim}{\phi^{0}}(\underset{\sim}{x})-\phi(\underset{\sim}{x})\right)}_{\sim})
$$

from Theorem C.1 of Appendix C an integration along $x_{3}$ leads to

$$
\left\|\underline{u}_{L}^{R M}-\underline{u}^{R M}\right\|_{H^{1}\left(P^{\varepsilon}\right)} \leq c \varepsilon^{3 / 2} .
$$

Collecting (45) and (47) we obtain

$$
\left\|\underline{u}^{\varepsilon}-\underline{u}_{L}\right\|_{H^{1}\left(P^{\varepsilon}\right)}+\left\|\underline{u}_{L}^{R M}-\underline{u}^{R M}\right\|_{H^{1}\left(P^{\varepsilon}\right)} \leq c \varepsilon^{3 / 2} .
$$

Furthermore, a lower bound for $\underline{u}^{\varepsilon}$ easily follows since

$$
\left\|\underline{u}^{\varepsilon}\right\|_{H^{1}\left(P^{\varepsilon}\right)} \geq\left\|u_{3}^{\varepsilon}\right\|_{H^{1}\left(P^{\varepsilon}\right)} \geq\left\|\zeta_{3}^{0}\right\|_{H^{1}\left(P^{\varepsilon}\right)}-\left\|u_{3}^{\varepsilon}-\zeta_{3}^{0}\right\|_{H^{1}\left(P^{\varepsilon}\right)} \geq c \varepsilon^{1 / 2},
$$

for $\varepsilon$ sufficiently small. Hence, from (44), (48) and (49) we have

$$
\frac{\left\|\underline{u}^{\varepsilon}-\underline{u}^{R M}\right\|_{H^{1}\left(P^{\varepsilon}\right)}}{\left\|\underline{u}^{\varepsilon}\right\|_{H^{1}\left(P^{\varepsilon}\right)}} \leq C \varepsilon
$$

Remark 3.3. From the proof of Theorem 3.2, it is easily seen that we have indeed

$$
\frac{\left\|{\underset{\sim}{\sim}}_{\sim}^{\varepsilon}-{\underset{\sim}{u}}^{R M}\right\|_{H^{1}\left(P^{\varepsilon}\right)}}{\left\|u_{\sim}^{\varepsilon}\right\|_{H^{1}\left(P^{\varepsilon}\right)}} \leq C \varepsilon, \quad \frac{\left\|u_{3}^{\varepsilon}-u_{3}^{R M}\right\|_{H^{1}\left(P^{\varepsilon}\right)}}{\left\|u_{3}^{\varepsilon}\right\|_{H^{1}\left(P^{\varepsilon}\right)}} \leq C \varepsilon .
$$




\subsection{Other convergence results}

In this subsection we collect some results concerning the modeling error for the stress field. Since the proofs are rather involved, we postpone them to Appendix D, for the sake of readability. We begin by recalling that $\underline{\underline{\sigma}}^{\varepsilon}$ is the 3D stress solution ( $c f$. (1)), while $\sigma^{R M}$ is the model stress solution recovered by (31).

The next Theorem gives an error estimate for the planar stress components.

Theorem 3.4. There exists a constant $C=C(\Omega, \underline{f}, \underline{g})$ independent of $\varepsilon$, such that

$$
\frac{\left\|\sigma_{\approx}^{\varepsilon}-{\underset{\sim}{\sigma}}^{\sigma^{R M}}\right\|_{L^{2}\left(P^{\varepsilon}\right)}}{\left\|\sigma_{\sim}^{\varepsilon}\right\|_{L^{2}\left(P^{\varepsilon}\right)}} \leq C \varepsilon^{1 / 2} .
$$

Regarding the convergence of the shear stress, we could obtain weaker results. In particular, due to boundary layer effects, only interior estimates have been developed. Nevertheless, the following theorem shows $O(\varepsilon)$ convergence for the averaged shear stress.

Theorem 3.5. Let $\Omega_{0}$ be a domain such that $\bar{\Omega}_{0} \subset \Omega$. Then there exists a constant $C=C(\Omega, \Omega(\underline{f}, \underline{g})$ independent of $\varepsilon$, such that

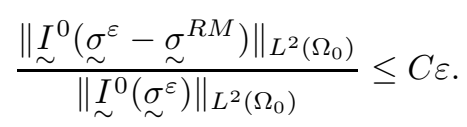

Theorem 3.5 can be used to obtain interior convergence estimates for the shear stress (not only for its average) in two particular situations.

The first one concerns the case of symmetric plates under bending, as displayed by the following corollary.

Corollary 3.6. Assume that $\nu$ and $E$ are even functions. Assume also that the loads induce a pure bending state, i.e.

- ${\underset{\sim}{f}}^{\varepsilon}$ is an odd function, and $f_{3}^{\varepsilon}$ is an even function, with respect to $x_{3}$;

- $\underline{g}^{\varepsilon}=\left(\underline{g}^{\varepsilon, \mathfrak{o}}, g_{3}^{\varepsilon, \mathfrak{e}}\right)$.

Let $\Omega_{0}$ be a domain such that $\bar{\Omega}_{0} \subset \Omega$, and set $P_{0}^{\varepsilon}=\Omega_{0} \times(-\varepsilon, \varepsilon)$. Then there exists a constant $C=C\left(\Omega, \Omega_{0}, \underline{f}, \underline{g}\right)$ independent of $\varepsilon$, such that

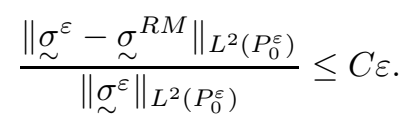

The second case is when the polynomials $p^{m}$ and $p^{b}$ are linearly dependent functions. For instance, this occurs whenever $\nu$ is constant. Indeed, we have the following result.

Corollary 3.7. Assume that $p^{m}$ and $p^{b}$ are linearly dependent functions. Let $\Omega_{0}$ be a domain such that $\bar{\Omega}_{0} \subset \Omega$, and set $P_{0}^{\varepsilon}=\Omega \times(-\varepsilon, \varepsilon)$. Then there exists a constant $C=C\left(\Omega, \Omega_{0}, \underline{f}, \underline{g}\right)$ independent of $\varepsilon$, such that

$$
\frac{\| \underset{\sim}{\sigma^{\varepsilon}-\sigma_{\sim}^{\sigma^{R M}} \|_{L^{2}\left(P_{0}^{\varepsilon}\right)}}}{\left\|\underset{\sim}{\sigma^{\varepsilon}}\right\|_{L^{2}\left(P_{0}^{\varepsilon}\right)}} \leq C \varepsilon .
$$

\section{Appendix A}

In this appendix we present the arguments necessary to obtain the model described in Section 2.3. Throughout this derivation, we assume that (21), (22) and (23) hold. Also, we consider that $p^{m}$ and $p^{b}$ are linearly independent. When these polynomials are linearly dependent, the computations are analogous and simpler. 
During the computations, we need that

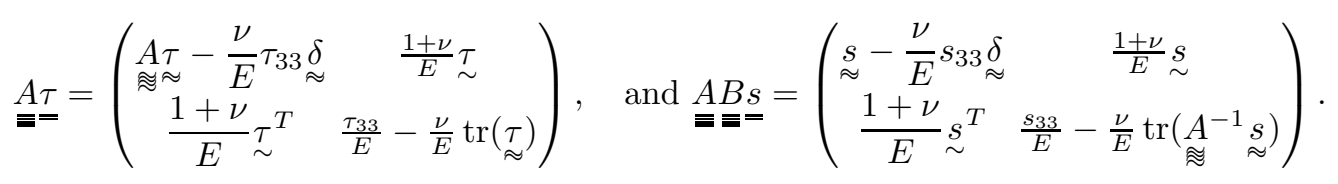

The equations (29), together with the boundary conditions for $\underset{\sim}{\eta}$ and $\underset{\sim}{\phi}$ in (26), are obtained from the first equation of (24), by considering

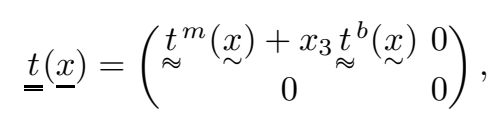

where $\underset{\sim}{t}, \underset{\sim}{t^{b}} \in \underset{\sim}{H}(\Omega)$ are arbitrary.

By considering next

$$
\left.\underline{\underline{t}}(\underline{x})=\left(\begin{array}{cc}
0 & \underset{\sim}{t^{m}}(\underset{\sim}{x}) p^{m}\left(x_{3}\right) \\
{\left[\underset{\sim}{t^{m}}(\underset{\sim}{x}) p^{m}\left(x_{3}\right)\right]^{T}} & 0
\end{array}\right), \quad \underline{\underline{t}} \underline{\underline{x}}\right)=\left(\begin{array}{cc}
0 & \underset{\sim}{t^{b}}(\underset{\sim}{x}) p^{b}\left(x_{3}\right) \\
{\left[\underset{\sim}{\left.t^{b}(\underset{\sim}{x}) p^{b}\left(x_{3}\right)\right]^{T}}\right.} & 0
\end{array}\right),
$$

in the first equation of (24), we conclude that

$$
\left\{\begin{array}{l}
\int_{-\varepsilon}^{\varepsilon} \frac{2(1+\nu)}{E}\left({\underset{\sim}{s}}^{m} p^{m}+{\underset{\sim}{s}}^{b} p^{b}\right) p^{m} \mathrm{~d} x_{3}=\int_{-\varepsilon}^{\varepsilon}(-\underset{\sim}{\phi}+\underset{\sim}{\nabla} \omega) p^{m} \mathrm{~d} x_{3}-\varepsilon^{2} \int_{-\varepsilon}^{\varepsilon} \frac{2(1+\nu)}{E} p_{\sim}^{l} p^{m} \mathrm{~d} x_{3}, \\
\int_{-\varepsilon}^{\varepsilon} \frac{2(1+\nu)}{E}\left({\underset{\sim}{s}}^{m} p^{m}+{\underset{\sim}{s}}^{b} p^{b}\right) p^{b} \mathrm{~d} x_{3}=\int_{-\varepsilon}^{\varepsilon}(-\underset{\sim}{\phi}+\underset{\sim}{\nabla} \omega) p^{b} \mathrm{~d} x_{3}-\varepsilon^{2} \int_{-\varepsilon}^{\varepsilon} \frac{2(1+\nu)}{E} \underset{\sim}{p} p^{b} \mathrm{~d} x_{3} .
\end{array}\right.
$$

Equation (30) expresses the solution of the above algebraic system. It follows also that $\omega=0$ on $\partial \Omega$.

Next, we consider the second equation of (24). By taking variations of test functions of the form $\underline{v}(\underline{x})=$ $(\underset{\sim}{v}(\underset{\sim}{x}), 0)$, we find

$$
\operatorname{div}_{\sim} \int_{-\varepsilon}^{\varepsilon} A_{\approx}^{-1}\left(\underset{\sim}{s^{m}}+\varepsilon^{-1} x_{3} \underset{\approx}{s^{b}}\right) \mathrm{d} x_{3}=-\int_{-\varepsilon}^{\varepsilon} f^{\varepsilon} \mathrm{d} x_{3}-2 \underset{\sim}{g^{\varepsilon, \mathfrak{e}}}
$$

Using the first equation of (29), we obtain the first equation of (26). By considering $\underline{v}(\underline{x})=\left(x_{3} \underset{\sim}{v}(\underset{\sim}{x}), 0\right)$ we find

$$
\operatorname{div}_{\sim} \int_{-\varepsilon}^{\varepsilon} x_{3}{\underset{\approx}{\sim}}^{-1}\left({\underset{\sim}{s}}^{m}+\varepsilon^{-1} x_{3} \underset{\sim}{s^{b}}\right) \mathrm{d} x_{3}-\int_{-\varepsilon}^{\varepsilon}\left({\underset{\sim}{s}}^{m} p^{m}+\underset{\sim}{s} p^{b}\right) \mathrm{d} x_{3}=-\int_{-\varepsilon}^{\varepsilon} x_{3} f^{\varepsilon} \mathrm{d} x_{3}-\varepsilon^{2} \int_{-\varepsilon}^{\varepsilon} x_{3} \frac{\partial \underline{p}^{l}}{\partial x_{3}} \mathrm{~d} x_{3},
$$

where we integrated by parts the second integral above. Using (30) we obtain that

$$
\stackrel{\sim}{\sim}^{m} I^{0}\left(p^{m}\right)+{\underset{\sim}{s}}^{b} I^{0}\left(p^{b}\right)=C^{S}(-\underset{\sim}{\phi}+\underset{\sim}{\nabla} \omega)-\varepsilon^{2}{\underset{\sim}{5}}_{5}
$$

Therefore, the second equation of (26) follows from (53), the second equation of (29) and from (54).

To obtain the final equilibrium equation, we consider $\underline{v}(\underline{x})=\left(0,0, v_{3}(\underset{\sim}{x})\right)$. Hence, it follows that

$$
\operatorname{div} \int_{-\varepsilon}^{\varepsilon}\left({\underset{\sim}{s}}^{m} p^{m}+{\underset{\sim}{s}}^{b} p^{b}\right) \mathrm{d} x_{3}=-\varepsilon^{3} l_{4}-\varepsilon^{2} \operatorname{div} \int_{-\varepsilon}^{\varepsilon} \underset{\sim}{p^{l}} \mathrm{~d} x_{3}
$$

Using then (54), we obtain the third equation of (26). 


\section{Appendix B}

As we mention in the introduction, to find out the suitable profiles for the shear stress, and also to derive error estimates, we look at the asymptotic expansion for the exact solution $\underline{u}^{\varepsilon}$. We do not give here a complete description of the expansion, but we only mention the basic ideas and some final results. This expansion for heterogeneous plates generalize the work of Dauge and Gruais [8]. See also [11], where the asymptotic for a general elasticity problem is investigated. The first step to obtain an asymptotic expansion for $\underline{u}^{\varepsilon}$ is, as usual, to introduce the change of variable $\left(\underset{\sim}{x}, x_{3}\right) \rightarrow\left(\underset{\sim}{x}, \hat{x}_{3}\right)=\left(\underset{\sim}{x}, \varepsilon^{-1} x_{3}\right)$, which maps the domain $P^{\varepsilon^{-}}=\Omega \times(-\varepsilon, \varepsilon)$ onto $\Omega \times(-1,1)$. Thus, the $3 \mathrm{D}$ elasticity problem

$$
\left\{\begin{array}{lr}
\left.-\underline{\operatorname{div}} \underline{A}^{-1} \underline{\underline{e}} \underline{\underline{\underline{u}}} \underline{u}^{\varepsilon}\right)=\underline{f}^{\varepsilon} & \text { in } P^{\varepsilon} \\
\underline{u}^{\varepsilon}=0 & \text { on } \partial P_{L}^{\varepsilon} \\
\underline{A}^{-1} \underline{\underline{e}}\left(\underline{u}^{\varepsilon}\right) \underline{n}=\underline{g}^{\varepsilon} & \text { on } \partial P_{ \pm}^{\varepsilon}
\end{array}\right.
$$

can be accordingly transformed into a problem (depending on the parameter $\varepsilon$ ) defined on the fixed domain $\Omega \times(-1,1)$. By assuming that:

- there exist $\varepsilon$-independent functions $\underline{f}: \Omega \times(-1,1) \rightarrow \mathbb{R}^{3}$, and $\underline{g}: \Omega \times\{-1,1\} \rightarrow \mathbb{R}^{3}$ such that

$$
\begin{cases}\underset{\sim}{f}\left(\underset{\sim}{x}, \hat{x}_{3}\right)=\varepsilon^{-1}{\underset{\sim}{f}}^{\varepsilon}\left(\underset{\sim}{x}, \varepsilon \hat{x}_{3}\right), & f_{3}\left(\underset{\sim}{x}, \hat{x}_{3}\right)=\varepsilon^{-2} f_{3}^{\varepsilon}\left(\underset{\sim}{x}, \varepsilon \hat{x}_{3}\right), \\ \left.\underset{\sim}{g} \underset{\sim}{x}, \hat{x}_{3}\right)=\varepsilon^{-2}{\underset{\sim}{q}}^{\varepsilon}\left(\underset{\sim}{x}, \varepsilon \hat{x}_{3}\right), & g_{3}\left(\underset{\sim}{x}, \hat{x}_{3}\right)=\varepsilon^{-3} g_{3}^{\varepsilon}\left(\underset{\sim}{x}, \varepsilon \hat{x}_{3}\right),\end{cases}
$$

for $\hat{x}_{3} \in(-1,1)$;

- the functions

$$
\hat{\nu}\left(\hat{x}_{3}\right)=\nu\left(\varepsilon \hat{x}_{3}\right), \quad \hat{E}\left(\hat{x}_{3}\right)=E\left(\varepsilon \hat{x}_{3}\right) \quad \text { for } \hat{x}_{3} \in(-1,1)
$$

are independent of $\varepsilon$;

an asymptotic expansion for $\underline{u}^{\varepsilon}$ reads as follows

$$
\begin{aligned}
& \underline{u}^{\varepsilon}(\underline{x}) \sim\left(\begin{array}{c}
\varepsilon \underbrace{\zeta^{1}}_{\sim}(\underset{\sim}{x})-x_{3} \underset{\sim}{\nabla} \zeta_{3}^{0}(\underset{\sim}{x}) \\
\zeta_{3}^{0}(\underset{\sim}{x})
\end{array}\right)+\varepsilon\left(\begin{array}{c}
\varepsilon \underbrace{\zeta^{2}}_{\sim}(\underset{\sim}{x})-x_{3} \underset{\sim}{\nabla} \zeta_{3}^{1}\left(\stackrel{\sim}{\sim}_{\sim}^{x}\right) \\
\zeta_{3}^{1}(\underset{\sim}{x})
\end{array}\right)+\varepsilon^{2}\left(\begin{array}{c}
0 \\
\stackrel{\sim}{u}_{3}^{2}\left(\underset{\sim}{x}, \varepsilon^{-1} x_{3}\right)
\end{array}\right)
\end{aligned}
$$

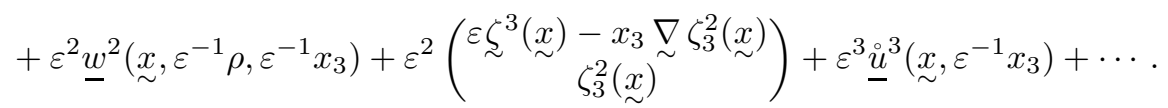

Above, $\underline{\zeta}^{k}: \Omega \rightarrow \mathbb{R}^{2}, \zeta_{3}^{k}: \Omega \rightarrow \mathbb{R}$ and $\underline{u}^{k}: \Omega \times(-1,1) \rightarrow \mathbb{R}^{3}$ are functions independent of $\varepsilon$. Moreover, the boundary correctors $\underline{w}^{k}$, needed to capture the boundary layers of the $3 \mathrm{D}$ solution, are functions which decay exponentially to zero with $\varepsilon^{-1} \rho$, where $\rho$ is the distance of a point $\underset{\sim}{x} \in \Omega$ from the boundary $\partial \Omega$. Inserting the formal expansion (57) into (55), one recognizes that the functions $\stackrel{\sim}{\zeta}^{k+1}$ and $\zeta_{3}^{k}$ can be determined by solving 2D partial differential equations on $\Omega$. In particular, the functions ${\underset{\sim}{\zeta}}^{1}$ and $\zeta_{3}^{0}$ entering in the leading term

$$
\underline{u}_{L}(\underline{x})=\left(\begin{array}{c}
\varepsilon{\underset{\sim}{(}}^{1}(\underset{\sim}{x})-x_{3} \underset{\sim}{\nabla} \zeta_{3}^{0}(\underset{\sim}{x}) \\
\zeta_{3}^{0}(\underset{\sim}{x})
\end{array}\right)
$$


of the expansion (57) solve the problem

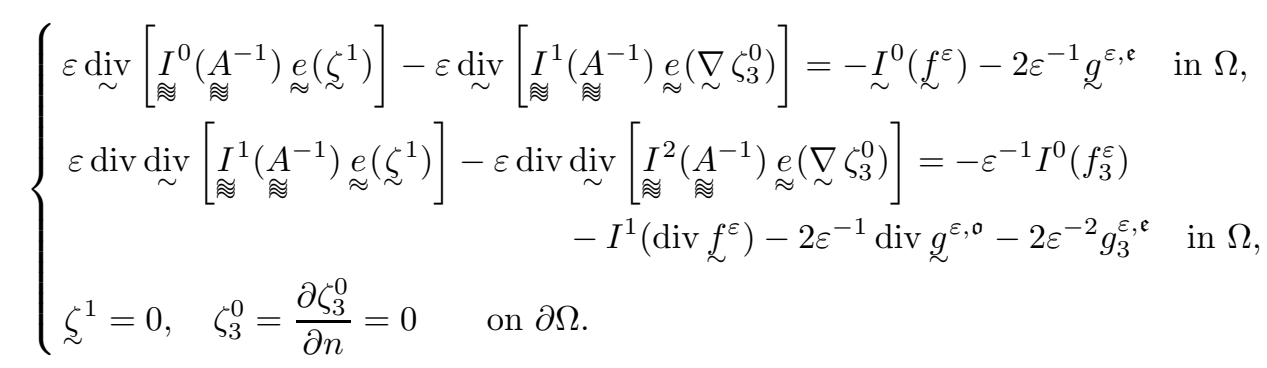

Furthermore the functions $\dot{u}^{k}$ are defined by solving a Neuman problem in each vertical fiber, i.e., these problems are posed in the one- $\overline{d i m e n s i o n a l ~ d o m a i n ~}(-1,1)$, and are parametrized by $\underset{\sim}{x} \in \Omega$. These functions are $\varepsilon$-independent and satisfy

$$
\int_{-1}^{1} \underline{\stackrel{\circ}{u}}^{k}\left(\underset{\sim}{x}, \hat{x}_{3}\right) \mathrm{d} \hat{x}_{3}=0 .
$$

The asymptotic expansion for the stress comes from formal substitution of (57) in the constitutive equation $\underline{\underline{\sigma^{\varepsilon}}}=\underline{\underline{\underline{A}}}^{-1} \underline{\underline{e}}\left(\underline{u}^{\varepsilon}\right)$. Hence

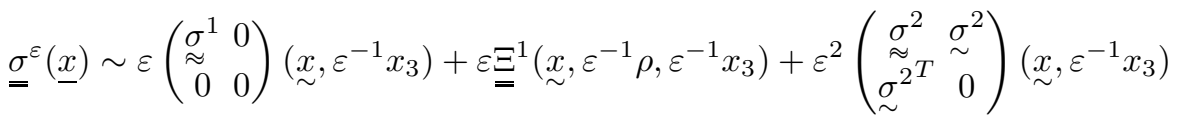

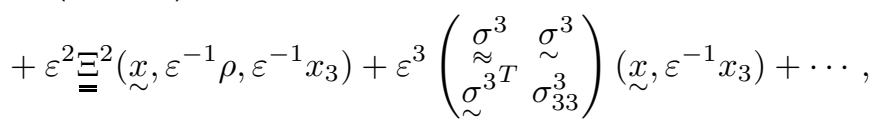

where $\underset{\Xi}{\Xi}$ correspond to boundary layer terms, and $\underset{=}{\sigma^{k}}$ are defined in $\Omega \times(-1,1)$. It can be shown that the components of $\underline{\sigma}^{k}$ are determined by

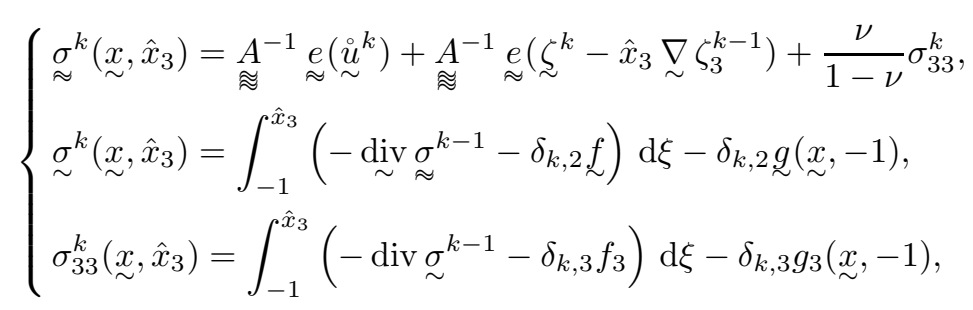

where $\delta_{k, l}$ denotes the Kronecker symbol. In particular, from (60) it follows

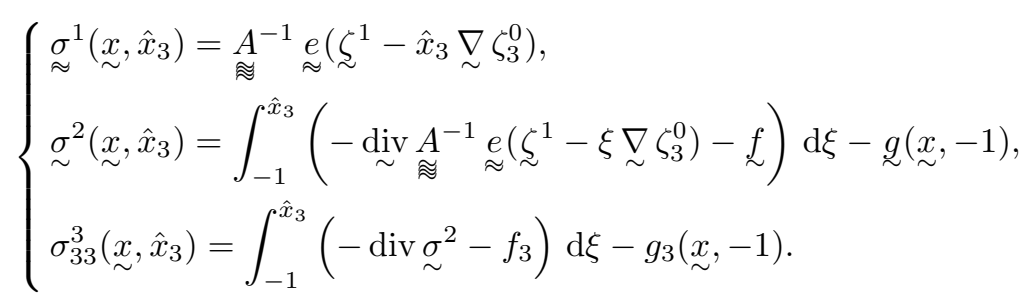

Although the first term of the asymptotic expansion of $\underset{\sim}{\sigma}$ is given by (61), the first equation of (59) can be used to provide an alternative expression of $\underset{\sim}{\sigma^{2}}$. More precisely, after an easy but cumbersome calculation, we find that

$$
{\underset{\sim}{\sigma}}^{2}\left(\underset{\sim}{x}, \hat{x}_{3}\right)=\underset{\sim}{\nabla} \operatorname{div} \underset{\sim}{\zeta^{1}}(\underset{\sim}{x}) p^{m}\left(\varepsilon \hat{x}_{3}\right)+\underset{\sim}{\nabla} \Delta \zeta_{3}^{0}(\underset{\sim}{x}) p^{b}\left(\varepsilon \hat{x}_{3}\right)+\underset{\sim}{p}\left(\underset{\sim}{x}, \varepsilon \hat{x}_{3}\right)
$$


where $(c f .(20))$

$$
\left\{\begin{array}{l}
p^{m}\left(x_{3}\right)=\frac{I^{0}\left(\frac{E \nu}{1-\nu^{2}}\right)}{I^{0}\left(\frac{E}{1+\nu}\right)} \varepsilon^{-1} \int_{-\varepsilon}^{x_{3}} \frac{E}{1+\nu} \mathrm{d} \xi-\varepsilon^{-1} \int_{-\varepsilon}^{x_{3}} \frac{E \nu}{1-\nu^{2}} \mathrm{~d} \xi, \\
p^{b}\left(x_{3}\right)=\frac{I^{1}\left(\frac{E}{1-\nu^{2}}\right)}{I^{0}\left(\frac{E}{1+\nu}\right)} \varepsilon^{-1} \int_{-\varepsilon}^{x_{3}} \frac{E}{1+\nu} \mathrm{d} \xi-\varepsilon^{-2} \int_{-\varepsilon}^{x_{3}} \frac{E}{1-\nu^{2}} \xi \mathrm{d} \xi, \\
\underset{\sim}{p^{l}}\left(\underset{\sim}{x}, x_{3}\right)=\frac{\varepsilon^{-3}}{I^{0}\left(\frac{E}{1+\nu}\right)}\left(\int_{-\varepsilon}^{\varepsilon} f^{\varepsilon} \mathrm{d} \xi+2 \underset{\sim}{g^{\varepsilon, e}}\right) \int_{-\varepsilon}^{x_{3}} \frac{E}{1+\nu} \mathrm{d} \xi-\varepsilon^{-2} \int_{-\varepsilon}^{x_{3}} f^{\varepsilon} \mathrm{d} \xi-\varepsilon^{-2}{\underset{\sim}{\sim}}_{\sim}^{\varepsilon}(\underset{\sim}{x},-\varepsilon) .
\end{array}\right.
$$

Even though the expansion (57) is formal, it is possible to derive asymptotic error estimates using the technique of [8]. Considering the error function ( $c f$. also (58))

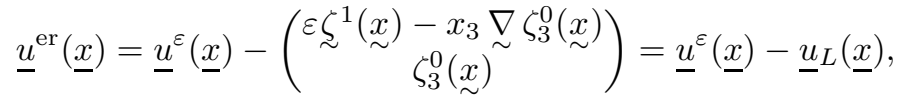

$$
\begin{aligned}
& \underline{\underline{\sigma}}^{\mathrm{er}}(\underline{x})=\underline{\sigma}^{\varepsilon}(\underline{x})-\left(\begin{array}{cc}
\varepsilon \underset{\sigma^{1}}{\sigma^{2}} & \varepsilon^{2} \sigma^{2} \\
\varepsilon^{2} \underset{\sim}{\sigma^{2}} & \varepsilon^{3} \sigma_{33}^{3}
\end{array}\right)\left(\underset{\sim}{x}, \varepsilon^{-1} x_{3}\right),
\end{aligned}
$$

one has the following result.

Theorem B.1. Given $\underline{u}^{\mathrm{er}}$ and $\underline{\underline{\sigma}}^{\mathrm{er}}$ as in (63), there exists a constant $C=C(\Omega, \underline{f}, \underline{g})$ independent of $\varepsilon$, such that

$$
\begin{gathered}
\left\|\underset{\sim}{u} u^{e r}\right\|_{H^{1}\left(P^{\varepsilon}\right)} \leq C \varepsilon^{2}, \quad\left\|u_{3}^{e r}\right\|_{H^{1}\left(P^{\varepsilon}\right)} \leq C \varepsilon^{3 / 2}, \\
\left\|{\underset{\sim}{\sigma}}^{e r}\right\|_{L^{2}\left(P^{\varepsilon}\right)} \leq C \varepsilon^{2}, \quad\left\|\sim_{\sim}^{e r}\right\|_{L^{2}\left(P^{\varepsilon}\right)} \leq C \varepsilon^{2}, \quad\left\|\sigma_{33}^{e r}\right\|_{L^{2}\left(P^{\varepsilon}\right)} \leq C \varepsilon^{2} .
\end{gathered}
$$

Moreover, let $\Omega_{0}$ be a domain such that $\bar{\Omega}_{0} \subset \Omega$, and let $P_{0}^{\varepsilon}=\Omega_{0} \times(-\varepsilon, \varepsilon)$. Then there exists a constant $C=C\left(\Omega, \Omega_{0}, \underline{f}, \underline{g}\right)$ independent of $\varepsilon$, such that

$$
\left\|u_{\sim}^{e r}\right\|_{H^{1}\left(P_{0}^{\varepsilon}\right)} \leq C \varepsilon^{5 / 2}, \quad\left\|\sigma_{\sim}^{e r}\right\|_{L^{2}\left(P_{0}^{\varepsilon}\right)} \leq C \varepsilon^{7 / 2}, \quad\left\|\sigma_{33}^{e r}\right\|_{L^{2}\left(P_{0}^{\varepsilon}\right)} \leq C \varepsilon^{9 / 2}
$$

\section{Appendix C}

In this Appendix we briefly report some results about the asymptotic expansion for the solution of our Reissner-Mindlin model (cf. (26)-(28)). We closely follow the arguments of Arnold and Falk [2], in which a detailed asymptotic analysis is provided for the bending problem of an isotropic and homogeneous plate.

The first step is to apply the divergence operator to the second equation of (26) and subtract the third equation of (26) from the result, to obtain

$$
\Delta \operatorname{div} \underset{\sim}{\phi}=\frac{1}{I^{2}(D)}\left[\varepsilon^{-1} I^{1}(D) \Delta \operatorname{div} \underset{\sim}{\eta}-\operatorname{div} \underset{\sim}{l} l_{2}+l_{4}-\varepsilon^{2} \operatorname{div} \underset{\sim}{l}\right],
$$

where $D=E /\left(1-\nu^{2}\right)$. Applying the Laplace operator to the third equation of (26) and using (67), we get

$$
\varepsilon I^{2}(D) \Delta^{2} \omega-I^{1}(D) \Delta \operatorname{div} \underset{\sim}{\eta}=\varepsilon\left(l_{4}-\operatorname{div} \underset{\sim}{l_{2}}\right)-\varepsilon^{3} \operatorname{div}{\underset{\sim}{3}}_{3}+\frac{I^{2}(D)}{C^{S}} \varepsilon^{3}\left[-\Delta \operatorname{div} \underset{\sim}{I^{0}}\left({\underset{\sim}{p}}^{l}\right)+\Delta \operatorname{div}{\underset{\sim}{l}}_{5}+\Delta l_{4}\right] .
$$


Above, the function $\underset{\sim}{p}$, the constant $C^{S}$ and the $l_{i}$ s are defined by (20), (27) and (28). Next, we assume the following formal expansions

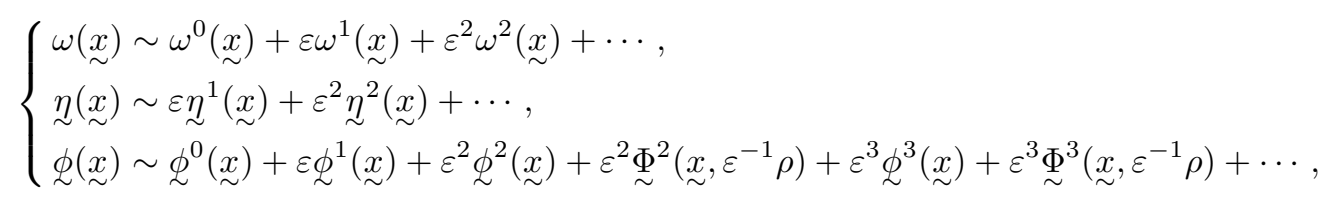

where $\underset{\sim}{\Phi^{i}}$ are boundary corrector terms. Note that there is no boundary layer for $\omega$ or $\underset{\sim}{ }$.

We also remark that, due to the choice (21), the expansion (69) implies that for the model displacement solution $\underline{u}^{R M}(\underline{x})$ we are supposing that

$$
\underline{u}^{R M}(\underline{x})=\underline{u}_{L}^{R M}(\underline{x})+\text { higher order terms }
$$

where

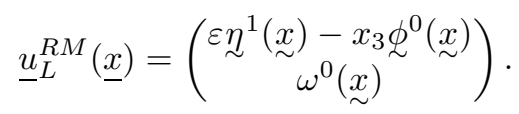

Disregarding the boundary correctors for $\underset{\sim}{\phi}$, formally substituting the above expansions in (26) and matching the terms with the same power of $\varepsilon$, we find that $\omega^{i},{\underset{\sim}{\sim}}^{i}$ and $\phi^{i}$ solve

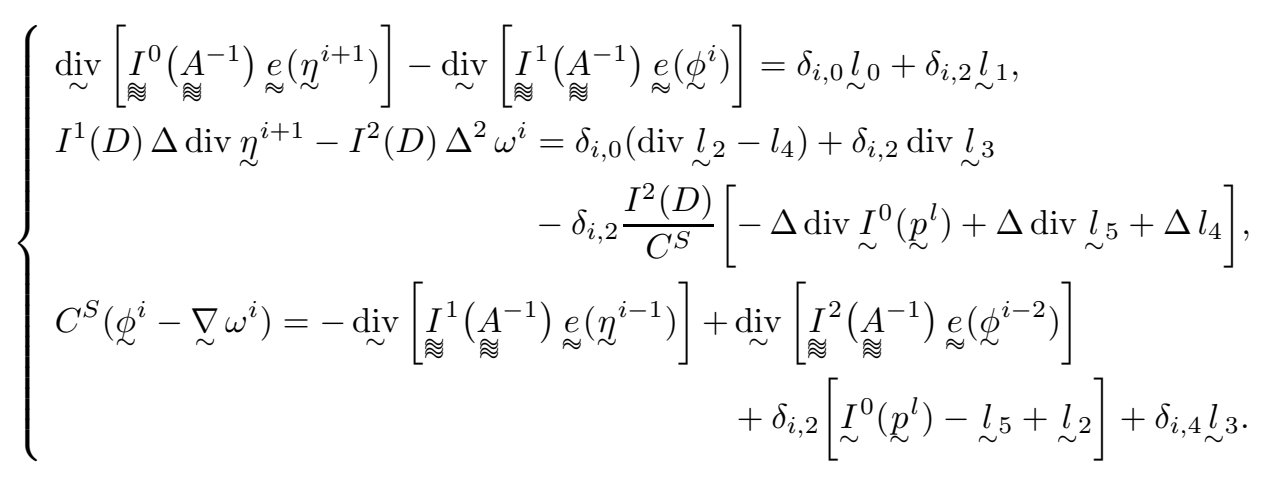

In particular, by taking $i=0$ in $(72)$, we have that $\eta^{1}, \phi^{0}$, and $\omega^{0}$ satisfy

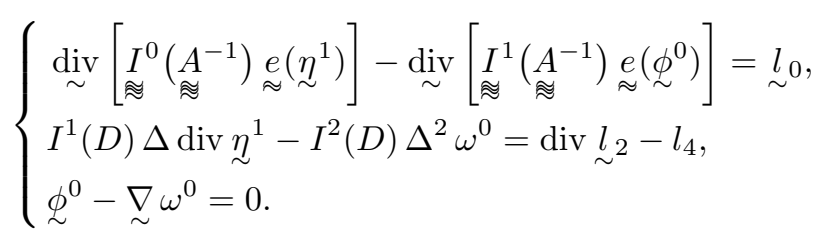

Recalling that $D=E /\left(1-\nu^{2}\right)$ and $(c f .(8))$

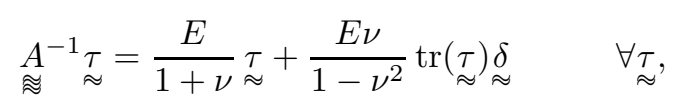


it is easily seen that (73) can be alternatively written as

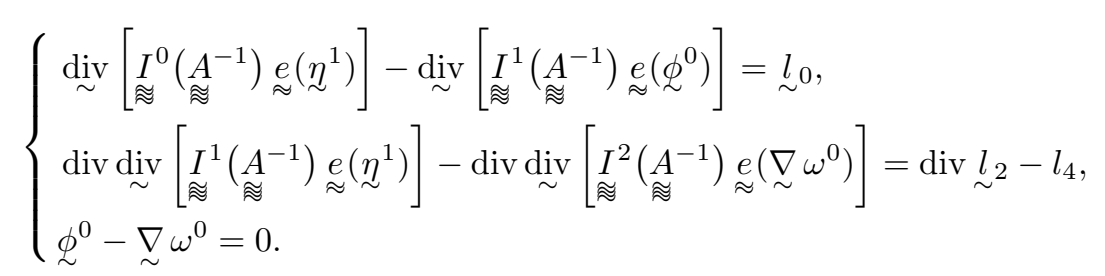

It is possible to estimate the difference between the leading terms in (69) and the exact Reissner-Mindlin solution, as stated in the theorem below. The basic ideas behind such proof is to first use (72) to obtain regularity estimates for the individual terms in the expansions (69), and then substitute a truncated expansion in the original system (26). Regularity results for (26) imply that adding more terms in the asymptotic series leads to smaller errors, by construction of the expansion. This is done in details, albeit for a simpler version of Reissner-Mindlin system, in [2].

Theorem C.1. Let $\underset{\sim}{\eta}, \underset{\sim}{\phi}$ and $\omega$ be the solution of (26). Assume that ${\underset{\sim}{\eta}}^{1},{\underset{\sim}{\phi}}^{0}$, and $\omega^{0}$ solve (73). Then, for every positive integer $\tilde{k}$, there exists a constant $C=C(\Omega, \underline{f}, \underline{g}, k)$ independent of $\varepsilon$ such that

$$
\varepsilon^{-1}\left\|\underset{\sim}{\eta}-\varepsilon{\underset{\sim}{\eta}}^{1}\right\|_{H^{k}(\Omega)}+\left\|\omega-\omega^{0}\right\|_{H^{k}(\Omega)}+\left\|\underset{\sim}{\phi}-{\underset{\sim}{0}}^{0}\right\|_{H^{1}(\Omega)} \leq C \varepsilon
$$

\section{Appendix D}

In this appendix we present proofs of the convergence results of Section 3.2.

Proof of Theorem 3.4. We recall that we wish to prove the estimate $\left\|\underset{\approx}{\sigma^{\varepsilon}}-\underset{\approx}{\sigma^{R M}}\right\|_{L^{2}\left(P^{\varepsilon}\right)} \leq C \varepsilon^{1 / 2} \|{\underset{\approx}{\sigma^{\varepsilon}}}_{L^{2}\left(P^{\varepsilon}\right)}$. We also recall that $(c f .(14)$ and $(31))$

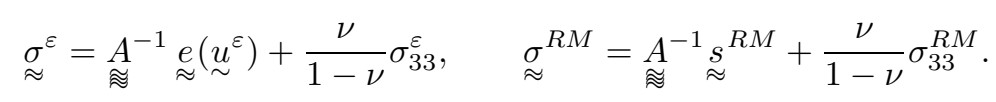

Thus, from the triangle inequality, we obtain

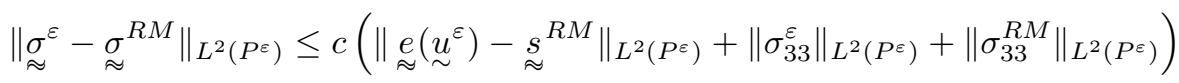

$$
\begin{aligned}
& \leq c\left(\left\|\underset{\sim}{e}\left(u_{\sim}^{\varepsilon}\right)-\underset{\sim}{e}\left(\varepsilon \underline{\sim}^{1}-x_{3} \underset{\sim}{\nabla} \zeta_{3}^{0}\right)\right\|_{L^{2}\left(P^{\varepsilon}\right)}+\left\|{\underset{\sim}{s}}^{R M}-\underset{\sim}{e}\left(\varepsilon{\underset{\sim}{\zeta}}^{1}-x_{3} \underset{\sim}{\nabla} \zeta_{3}^{0}\right)\right\|_{L^{2}\left(P^{\varepsilon}\right)}\right. \\
& \left.+\left\|\sigma_{33}^{\varepsilon}\right\|_{L^{2}\left(P^{\varepsilon}\right)}+\left\|\sigma_{33}^{R M}\right\|_{L^{2}\left(P^{\varepsilon}\right)}\right) \text {. }
\end{aligned}
$$

From the two-dimensional Korn's inequality in $\Omega$ and the first estimate of (65), we have

$$
\left\|\underset{\approx}{e}\left(u_{\sim}^{\varepsilon}\right)-\underset{\approx}{e}\left(\varepsilon \zeta^{1}-x_{3} \underset{\sim}{\nabla} \zeta_{3}^{0}\right)\right\|_{L^{2}\left(P^{\varepsilon}\right)} \leq c \varepsilon^{2} .
$$

From (65) of Theorem B.1, the estimate $\left\|\sigma_{33}^{3}\right\|_{L^{2}\left(P^{\varepsilon}\right)} \leq c \varepsilon^{1 / 2}$, it follows that

$$
\left\|\sigma_{33}^{\varepsilon}\right\|_{L^{2}\left(P^{\varepsilon}\right)} \leq\left\|\sigma_{33}^{\varepsilon}-\varepsilon^{3} \sigma_{33}^{3}\right\|_{L^{2}\left(P^{\varepsilon}\right)}+\varepsilon^{3}\left\|\sigma_{33}^{3}\right\|_{L^{2}\left(P^{\varepsilon}\right)} \leq c \varepsilon^{2} .
$$

Next, from the definition of $\sigma_{33}^{R M}$ in (31), (9), and the scaling assumption (6), we have

$$
\left\|\sigma_{33}^{R M}\right\|_{L^{2}\left(P^{\varepsilon}\right)} \leq c \varepsilon^{2} .
$$


To bound the term $\left\|\underset{\sim}{s^{R M}}-\underset{\sim}{e}\left(\varepsilon{\underset{\sim}{\eta}}^{1}-x_{3} \underset{\sim}{\nabla} \zeta_{3}^{0}\right)\right\|_{L^{2}\left(P^{\varepsilon}\right)}$, we first recall that $(c f .(22)) \underset{\sim}{\underset{\sim}{\eta^{1}}}{ }^{R M}=\underset{\sim}{s^{m}}+\varepsilon^{-1} x_{3} \underset{\sim}{s^{b}}$, where $\left(\stackrel{\sim}{\sim}^{m},{\underset{\sim}{s}}^{b}\right)$ is the solution of system $(29)$. Therefore, since Lemma 3.1 implies $\underset{\sim}{\zeta}={\underset{\sim}{\eta}}^{1}$ and $\underset{\sim}{\nabla} \zeta_{3}^{0}=\dot{\sim}^{0}$, we get

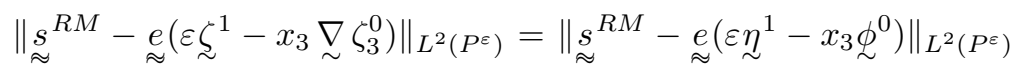

$$
\begin{aligned}
& \leq\left\|{\underset{\sim}{s}}^{m}-\varepsilon \underset{\sim}{e}\left(\eta^{1}\right)\right\|_{L^{2}\left(P^{\varepsilon}\right)}+\left\|\varepsilon^{-1} x_{3}\left(\underset{\sim}{s^{b}}+\varepsilon \underset{\sim}{e}\left(\phi^{0}\right)\right)\right\|_{L^{2}\left(P^{\varepsilon}\right)} .
\end{aligned}
$$

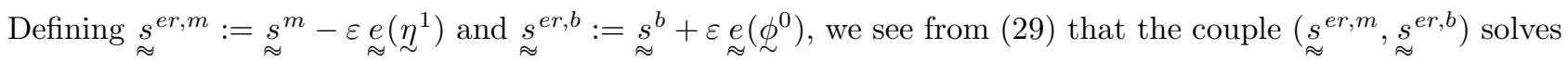

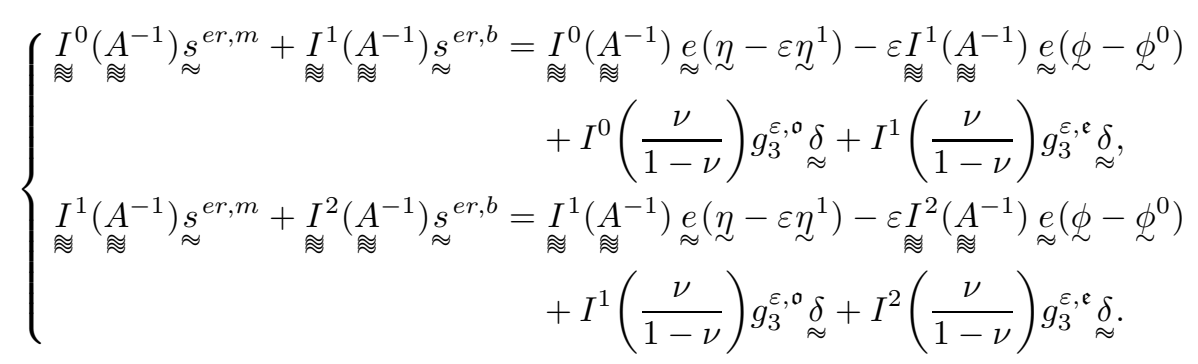

Therefore, for every $\underset{\sim}{x} \in \Omega$, we have

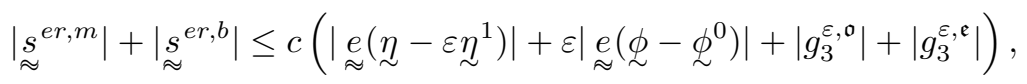

where $|\cdot|$ denotes the usual algebraic norm for tensors. Hence, using (6) and Theorem C.1 we get

$$
\left\|{\underset{\sim}{s}}^{e r, m}\right\|_{L^{2}\left(P^{\varepsilon}\right)}+\left\|{\underset{\sim}{s}}^{e r, b}\right\|_{L^{2}\left(P^{\varepsilon}\right)} \leq \varepsilon^{5 / 2} .
$$

Collecting (76)-(81) we obtain

$$
\left\|\left.\right|_{\approx} ^{\varepsilon}-\underset{\approx}{\sigma^{R M}}\right\|_{L^{2}\left(P^{\varepsilon}\right)} \leq \varepsilon^{2} .
$$

Moreover, proceeding as in (49) we find the following lower bound for $\underset{\sim}{\sigma^{\varepsilon}}$

$$
\left\|\sigma_{\approx}^{\varepsilon}\right\|_{L^{2}\left(P^{\varepsilon}\right)} \geq \varepsilon\left\|\sigma_{\approx}^{1}\right\|_{L^{2}\left(P^{\varepsilon}\right)}-\left\|\sigma_{\approx}^{\varepsilon}-\varepsilon \sigma^{1}\right\|_{L^{2}\left(P^{\varepsilon}\right)} \geq c \varepsilon^{3 / 2}
$$

for $\varepsilon$ sufficiently small. The theorem now follows from (82) and (83).

Proof of Theorem 3.5. We wish to obtain $\left\|{\underset{\sim}{I}}^{0}\left({\underset{\sim}{\sigma}}^{\varepsilon}-{\underset{\sim}{\sigma}}^{R M}\right)\right\|_{L^{2}\left(\Omega_{0}\right)} \leq C \varepsilon\left\|{ }_{\sim}{ }^{0}\left({\underset{\sim}{\sigma}}^{\varepsilon}\right)\right\|_{L^{2}\left(\Omega_{0}\right)}$. We first recall that (cf. (31) and (23))

$$
{\underset{\sim}{\sigma}}^{R M}=\varepsilon^{2} \underline{\sim}^{l}(\underline{x})+\underset{\sim}{s}{ }_{\sim}^{m}(\underset{\sim}{x}) p^{m}\left(x_{3}\right)+\underset{\sim}{s}(\underset{\sim}{b}) p^{b}\left(x_{3}\right) .
$$

We focus on the more difficult case of $p^{m}$ and $p^{b}$ linearly independent (otherwise the computations are easier).

From the triangle inequality it follows that

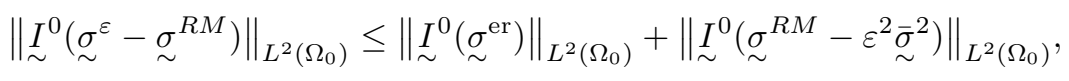

where $\underset{\sim}{\text { er }}$ is defined in $(63)$ and $\underset{\sim}{\bar{\sigma}^{2}}(\underline{x})=\underset{\sim}{\sigma^{2}}\left(\underset{\sim}{x}, \varepsilon^{-1} x_{3}\right)$. Using Cauchy-Schwarz inequality, we find that

$$
\left\|\varepsilon I_{\sim}^{0}\left(\sigma_{\sim}^{\mathrm{er}}\right)\right\|_{L^{2}\left(\Omega_{0}\right)} \leq(2 \varepsilon)^{1 / 2}\left\|\left(\int_{-\varepsilon}^{\varepsilon}\left|\sigma_{\sim}^{\mathrm{er}}\right|^{2} \mathrm{~d} x_{3}\right)^{1 / 2}\right\|_{L^{2}\left(\Omega_{0}\right)}=(2 \varepsilon)^{1 / 2}\left\|\sigma_{\sim}^{\mathrm{er}}\right\|_{L^{2}\left(P_{0}^{\varepsilon}\right)} \leq c \varepsilon^{4},
$$


where Theorem B.1 is used to obtain the last inequality (cf. (66)). Using (53) and (29), we have that

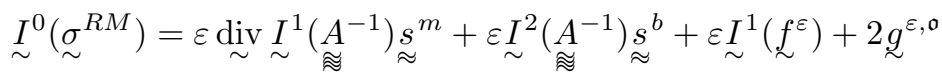

$$
\begin{aligned}
& =\varepsilon \operatorname{div}_{\sim} \underset{\sim}{I^{1}}\left(\underset{\approx}{A^{-1}}\right) \underset{\sim}{e}(\eta)-\varepsilon^{2} \operatorname{div}_{\sim} \underset{\sim}{I^{2}}\left(\underset{\approx}{A^{-1}}\right) \underset{\sim}{e}(\phi)+\varepsilon I^{1}\left(\frac{\nu}{1-\nu}\right) \underset{\sim}{\nabla} g_{3}^{\varepsilon, \mathfrak{o}} \\
& +\varepsilon I^{2}\left(\frac{\nu}{1-\nu}\right) \underset{\sim}{\nabla} g_{3}^{\varepsilon, \mathfrak{e}}+\varepsilon \underset{\sim}{I^{1}}\left({\underset{\sim}{\varepsilon}}^{\varepsilon}\right)+2 g_{\sim}^{\varepsilon, \mathfrak{o}} .
\end{aligned}
$$

Using (61) and integrating by parts, we gather that

$$
\begin{aligned}
& \int_{-\varepsilon}^{\varepsilon} \underset{\sim}{\sigma^{2}}\left(\cdot, \varepsilon^{-1} x_{3}\right) \mathrm{d} x_{3}=\int_{-\varepsilon}^{\varepsilon}\left(x_{3}^{\prime} \int_{-1}^{\varepsilon^{-1} x_{3}}\left(-\operatorname{div}_{\sim} \underset{\approx}{A^{-1}} \underset{\approx}{e}\left(\zeta^{1}-\xi \underset{\sim}{\nabla} \zeta_{3}^{0}\right)-\underset{\sim}{f}\right) \mathrm{d} \xi-\underset{\sim}{g}(\underset{\sim}{x},-1)\right) \mathrm{d} x_{3} \\
& \left.=\int_{-\varepsilon}^{\varepsilon} \varepsilon^{-1} x_{3}\left[\underset{\sim}{\operatorname{div}} \underset{\approx}{A^{-1}} \underset{\sim}{e}\left(\zeta^{1}-\varepsilon^{-1} x_{3} \underset{\sim}{\nabla} \zeta_{3}^{0}\right)+\underset{\sim}{f}\left(\cdot, \varepsilon^{-1} x_{3}\right)\right] \mathrm{d} x_{3}-2 \underset{\sim}{g} \underset{\sim}{x},-1\right) \\
& +\varepsilon \int_{-1}^{1}\left(-\operatorname{div}_{\sim} \underset{\approx}{A^{-1}} \underset{\approx}{e}\left(\zeta^{1}-\xi \underset{\sim}{\nabla} \zeta_{3}^{0}\right)-\underset{\sim}{f}\right) \mathrm{d} \xi .
\end{aligned}
$$

Substituting the first equation of (7) in (86), we obtain

$$
{\underset{\sim}{I}}^{0}\left(\bar{\sigma}^{2}\right)=\operatorname{div}_{\sim} I_{\approx}^{1}\left(\underset{\approx}{A^{-1}}\right) \underset{\approx}{e}\left(\zeta^{1}\right)+\operatorname{div}_{\sim} I_{\approx}^{2}\left(\underset{\approx}{A^{-1}}\right) \underset{\sim}{e}\left(\underset{\sim}{\nabla} \zeta_{3}^{0}\right)+\underset{\sim}{I^{1}}(\underset{\sim}{f})+2 \varepsilon^{-2} g^{\varepsilon, \mathfrak{o}} .
$$

Therefore, from (85) and (87) we get

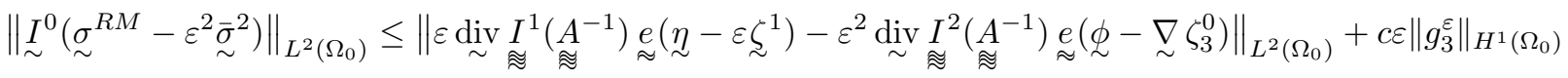

$$
\begin{aligned}
& \leq c \varepsilon\left\|\eta-\varepsilon \zeta^{1}\right\|_{H^{2}\left(\Omega_{0}\right)}+c \varepsilon^{2}\left\|\phi-\underset{\sim}{\nabla} \zeta_{3}^{0}\right\|_{H^{2}\left(\Omega_{0}\right)}+c \varepsilon\left\|g_{3}^{\varepsilon}\right\|_{H^{1}\left(\Omega_{0}\right)} \leq c \varepsilon^{3},
\end{aligned}
$$

where the final inequality follows from Theorem C.1 and Hypothesis (6). Combining estimates (84) and (88), we conclude that

$$
\left\|I_{\sim}^{0}\left({\underset{\sim}{\varepsilon}}^{\varepsilon}-{\underset{\sim}{\sigma}}^{R M}\right)\right\|_{L^{2}\left(\Omega_{0}\right)} \leq c \varepsilon^{3}
$$

The result follows since from Theorem B.1 and the definition of $\underset{\sim}{\sigma^{2}}$, it holds

$$
\left\|{\underset{\sim}{I}}^{0}\left(\underset{\sim}{\sigma^{\varepsilon}}\right)\right\|_{L^{2}\left(\Omega_{0}\right)} \geq c \varepsilon^{2}
$$

Proof of Corollary 3.6. Our aim is to prove that

$$
\frac{\left\|{\underset{\sim}{\sigma}}^{\varepsilon}-{\underset{\sim}{\sigma}}^{R M}\right\|_{L^{2}\left(P_{0}^{\varepsilon}\right)}}{\left\|\sigma_{\sim}^{\varepsilon}\right\|_{L^{2}\left(P_{0}^{\varepsilon}\right)}} \leq C \varepsilon .
$$

As before, let $\underset{\sim}{\bar{\sigma}^{2}}(\underline{x})=\underset{\sim}{\sigma^{2}}\left(\underset{\sim}{x}, \varepsilon^{-1} x_{3}\right)$. From the triangle inequality and Theorem B.1, we have

$$
\left\|\sigma_{\sim}^{\varepsilon}-{\underset{\sim}{\sigma}}^{R M}\right\|_{L^{2}\left(P_{0}^{\varepsilon}\right)} \leq\|\|_{\sim}^{\varepsilon}-\varepsilon^{2} \bar{\sim}^{2}\left\|_{L^{2}\left(P_{0}^{\varepsilon}\right)}+\right\| \sigma_{\sim}^{R M}-\varepsilon^{2} \bar{\sim}^{2}\left\|_{L^{2}\left(P_{0}^{\varepsilon}\right)} \leq\right\| \sigma_{\sim}^{R M}-\varepsilon^{2} \bar{\sigma}^{2} \|_{L^{2}\left(P_{0}^{\varepsilon}\right)}+c \varepsilon^{7 / 2} .
$$

In the pure bending case, $\sigma_{\sim}^{R M}$ simplifies as

$$
{\underset{\sim}{\sigma}}^{R M}={\underset{\sim}{s}}^{b} p^{b}+\varepsilon^{2}{\underset{\sim}{p}}^{l}
$$


Hence, from the definition of $\underset{\sim}{\bar{\sigma}^{2}}$ and $(62)$, we obtain

$$
\left\|{\underset{\sim}{I}}^{0}\left({\underset{\sim}{\sigma}}^{R M}-\varepsilon^{2} \bar{\sigma}^{2}\right)\right\|_{L^{2}\left(\Omega_{0}\right)}=\left\|\left({\underset{\sim}{s}}^{b}-\varepsilon^{2} \underset{\sim}{\nabla} \Delta \zeta_{3}^{0}\right) \underset{\sim}{I^{0}}\left(p^{b}\right)\right\|_{L^{2}\left(\Omega_{0}\right)}=\left\|{\underset{\sim}{s}}^{b}-\varepsilon^{2} \underset{\sim}{\nabla} \Delta \zeta_{3}^{0}\right\|_{L^{2}\left(\Omega_{0}\right)}\left|{\underset{\sim}{I}}^{0}\left(p^{b}\right)\right| .
$$

Using (88) we get

Thus, it follows that

$$
\left\|\stackrel{\sim}{s}^{b}-\varepsilon^{2} \underset{\sim}{\nabla} \Delta \zeta_{3}^{0}\right\|_{L^{2}\left(\Omega_{0}\right)} \leq c \varepsilon^{3}
$$

$$
\left\|\sigma_{\sim}^{R M}-\varepsilon^{2} \bar{\sim}^{2}\right\|_{L^{2}\left(P_{0}^{\varepsilon}\right)}=\left\|p^{b}\right\|_{L^{2}(-\varepsilon, \varepsilon)}\left\|{\underset{\sim}{s}}^{b}-\varepsilon^{2} \underset{\sim}{\nabla} \Delta \zeta_{3}^{0}\right\|_{L^{2}\left(\Omega_{0}\right)} \leq c \varepsilon^{7 / 2}
$$

and the result is a consequence of $\left\|{\underset{\sim}{\sigma}}^{\varepsilon}\right\|_{L^{2}\left(P_{0}^{\varepsilon}\right)} \geq c \varepsilon^{5 / 2}$.

Proof of Corollary 3.7. We recall that we are considering the case of $p^{m}$ and $p^{b}$ linearly dependent. Therefore, let $a \in \mathbb{R}$ such that $p^{m}=a p^{b}$. Then, using the same notation of the proof of Theorem 3.6, we have that

$$
\stackrel{\bar{\sigma}}{\sim}^{2}=\left(a \underset{\sim}{\nabla} \operatorname{div} \underset{\sim}{\zeta^{1}}+\underset{\sim}{\nabla} \Delta \zeta_{3}^{0}\right) p^{b}
$$

It is enough now to proceed as in the proof of Theorem 3.6.

\section{REFERENCES}

[1] S.M. Alessandrini, D.N. Arnold, R.S. Falk and A.L. Madureira, Derivation and Justification of Plate Models by Variational Methods. Centre de Recherches Mathematiques, CRM Proceedings and Lecture Notes (1999).

[2] D.N. Arnold and R.S. Falk, Asymptotic analysis of the boundary layer for the Reissner Mindlin plate model. SIAM J. Math. Anal. 27 (1996) 486-514.

[3] D.N. Arnold, A.L. Madureira and S. Zhang, On the range of applicability of the Reissner-Mindlin and Kirchhoff-Love plate bending models. J. Elasticity 67 (2002) 171-185.

[4] F. Auricchio and E. Sacco, Partial-mixed formulation and refined models for the analysis of composite laminates within FSDT. Composite Structures 46 (1999) 103-113.

[5] D. Caillerie, Thin elastic and periodic plates. Math. Meth. Appl. Sci. 6 (1984) 159-191.

[6] P.G. Ciarlet, Mathematical Elasticity, volume II: Theory of Plates, North-Holland Publishing Co., Amsterdam. Stud. Math. Appl. 27 (1997)

[7] P.G. Ciarlet and Ph. Destuynder, A justification of the two dimensional linear plate model. J. Mécanique 18 (1979) 315-344.

[8] M. Dauge and I. Gruais, Asymptotics of arbitrary order for a thin elastic clamped plate, I. Optimal error estimates. Asymptotic Anal. 13 (1996) 167-197.

[9] P. Destuynder, Sur une justification des modèles de plaques et de coques par les méthodes asymptotiques. Ph.D. thesis, Université Pierre et Marie Curie - Paris, France (1980).

[10] K.H. Lo, R.M. Christensen and E.M. Wu, A high-order theory of plate deformation. J. Appl. Mech. 46 (1977) $663-676$.

[11] O.V. Motygin and S.A. Nazarov, Justification of the Kirchhoff hypotheses and error estimation for two-dimensional models of anisotropic and inhomogeneous plates, including laminated plates. IMA J. Appl. Math. 65 (2000) 1-28.

[12] J.C. Paumier and A. Raoult, Asymptotic consistency of the polynomial approximation in the linearized plate theory application to the Reissner-Mindlin model. ESAIM: Proc. 2 (1997) 203-213.

[13] J. Sanchez Hubert and E. Sanchez Palencia, Introduction aux méthodes asymptotiques et à l'homogénéisation, Masson, Paris (1992). 